\title{
Direct estimation of multidimensional perceptual distributions: Assessing hue and form
}

\author{
DALE J. COHEN \\ University of North Carolina, Wilmington, North Carolina
}

\begin{abstract}
The procedures developed to assess the perceptual and decisional processes associated with detection in multidimensional space all require specialized statisticalskills and analysis programs. The present article describes a regression model, designed to assess dimensional interactions, that is both computationally simpler and more accessiblethan those procedures. The paper validatesthe regression model by comparing the perceptual space associated with the detection of hue and form mapped by the regression model with that mapped by Kadlec and Townsend's (1992a, 1992b) macro- and microanalyses. The results of both analyses showed that hue strongly influences the perception of form but that form only weakly influences the perception of hue. The parallel results of the two analyses suggest that the regression model is a valid alternative to multidimensional signal detection theory analysis.
\end{abstract}

All stimuli are multidimensional. Ashby and Townsend (1986) have made the point that "a fundamentally important problem is to determine how these dimensions are combined in perceptual processing" (p. 154). Toward that end, Ashby and Townsend proposed a general recognition theory (GRT), which extends signal detection theory (SDT) to multidimensional space. Unfortunately, assessing the perceptual and decisional processes associated with detection in multidimensional space is more complicated than equivalent assessments in unidimensional space (e.g., Ashby \& Townsend, 1986). The few procedures developed to assess these processes in multidimensional space all require specialized statistical skills and analysis programs (e.g., Ashby \& Lee, 1991; Kadlec \& Townsend, 1992a, 1992b; Maddox, 2001; Maddox \& Ashby, 1996; Wickens, 1992). It is perhaps because of this requirement that multidimensional stimulus detection has not received the attention afforded to unidimensional stimulus detection. In an effort to make multidimensional stimulus detection more accessible, the present article proposes and validates a relatively simple regression procedure that provides information about dimensional interaction similar to that provided by an established analytic procedure based on SDT (Kadlec $\&$ Townsend's, 1992a, 1992b, macro- and microanalyses).

\section{GENERAL RECOGNITION THEORY}

Perhaps the most widely recognized theory of dimensional interaction is GRT (Ashby \& Townsend, 1986). GRT assumes a multidimensional perceptual space, with the perceptual effect $(\Psi)$ of each dimension lying on separate

I thank S. Cohen for her helpful comments and support at various stages of this project. I also thank Helena Kadlec and two anonymous reviewers for their very helpful critiques. Correspondence concerning this article should be sent to D. J. Cohen, Department of Psychology, University of North Carolina, Wilmington, NC 28403 (e-mail: cohend@ uncw.edu). axes. For example, one may create a two-dimensional space with the perceptual effect of Dimension $\mathbf{A}$ on the $x$-axis and the perceptual effect of Dimension $\mathbf{B}$ on the $y$-axis (see Figure 1). A specific stimulus presentation is represented by a point in space that corresponds to the perceptual effect of each dimension on that presentation. GRT, like SDT, assumes that there is noise in the system. Therefore, a specific stimulus will give rise to different perceptual effects at each viewing. Multiple viewings of a stimulus will, therefore, produce a cloud of points, with each point landing in a region of space with a particular probability (called the perceptual distribution of the stimulus). The bivariate mean $\mu=\left[\mu_{\mathbf{A}}, \mu_{\mathbf{B}}\right]$ and the variance/ covariance matrix $\Sigma=\left[\sigma_{\mathbf{A}}^{2}, \operatorname{cov}_{\mathbf{A B}}, \sigma_{\mathbf{B}}^{2}\right]$ of the perceptual distribution summarize the perception of a particular stimulus along the dimensions assessed. ${ }^{1}$

To assess dimensional interaction, one must evaluate at least three of the four stimuli (i.e., $\mathbf{A}_{0} \mathbf{B}_{0}, \mathbf{A}_{1} \mathbf{B}_{0}, \mathbf{A}_{0} \mathbf{B}_{1}$, $\mathbf{A}_{1} \mathbf{B}_{1}$ ) displayed in Figure 1 (Tanner, 1956). These four stimuli are composed of a factorial combination of two dimensions ( $\mathbf{A}$ and $\mathbf{B})$, with each dimension composed of two values, $i=\{0,1\}$ and $j=\{0,1\}$. If two dimensions are independent, then (1) the feature value of one dimension will not affect the perception of the other dimension (i.e., $\mu_{\mathbf{A}_{i} \mathbf{B}_{0}}=\mu_{\mathbf{A}_{i} \mathbf{B}_{1}}$ and $\sigma_{\mathbf{A}_{i} \mathbf{B}_{0}}^{2}=\sigma_{\mathbf{A}_{i} \mathbf{B}_{1}}^{2}$, for all values of $i$ ), and (2) the perceptual noise on the two dimensions will be uncorrelated (Ashby \& Townsend, 1986). The former is a characteristic of the relation between stimuli and is termed perceptual separability. The latter is a characteristic of an individual stimulus and is termed perceptual independence. Figure 1 illustrates a variety of perceptual spaces. In Figure 1, perceptual separability holds for a dimension if (1) the dotted lines connecting the bivariate means of the perceptual distributions are perpendicular to that dimension's axis and (2) the equal likelihood contours of the perceptual distributions along that dimension are constant across the values of the other dimension. Perceptual inde- 

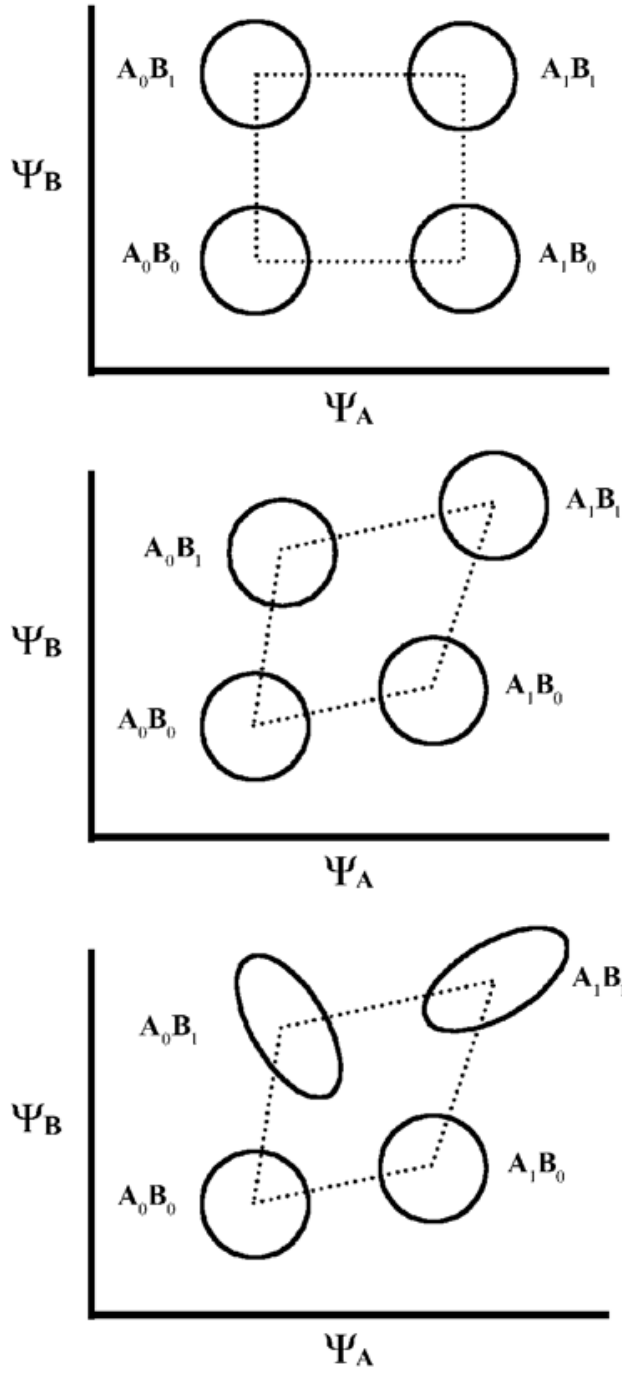

Figure 1. Three two-dimensional perceptual spaces. The dotted polygon connects the bivariate means of the perceptual distributions. The solid ellipses represent equal likelihood contours of the perceptual distributions. The top graph shows a perceptual space where both perceptual separability and perceptual independence hold. In the middle graph, perceptual separability fails for both dimensions, and perceptual independence holds. In the bottom graph, perceptual separability fails for both dimensions, and perceptual independence fails for two stimuli.

pendence holds if the major and the minor axes of the ellipse representing equal likelihood contours of the perceptual distribution are parallel to the perceptual axes (i.e., the "cloud" of points will be circular, or if elliptical, the orientation of the cloud of points should be vertical or horizontal).

In addition to modeling the perceptual space, GRT also models the response strategy of the participant. When making a response, the participant can judge the perceptual effect of each dimension separately, or his or her assessment of one dimension may affect his or her assessment of the other dimension. GRT terms an independent response strategy decisional separability. Decisional separability is represented in the perceptual space by decision criteria that are parallel to the perceptual axes.

\section{Processing Model}

Ashby (1989) instantiates GRT in a processing model that incorporates a separate processing stream for each dimension assessed. Each processing stream is divided into three consecutive stages in which dimensional interaction may occur. Figure 2 presents a version of that model.

Stage 1 is characterized by the sensitivity of each processing stream to various feature dimensions. A Stage 1 interaction occurs when the receptors of the stream processing Dimension $\mathbf{A}$ are also sensitive to variations in Dimension B. For example, if the receptors in the stream that is processing form are also somewhat sensitive to hue, variation in the hue dimension may affect the perception of the form dimension. Thus, Stage 1 interaction is an interaction between a single processing stream and the dimensions that make up the physical stimulus.

Stage 2 is characterized by communication between processing streams. Stage 2 interaction occurs when the activation of Stream $\mathbf{A}$ influences the activation of Stream $\mathbf{B}$. For example, the stream that is processing form may also be sensitive to the activation of the stream that is processing hue. In this instance, the stream that is processing hue mediates the influence of hue on the stream that is processing form. Thus, Stage 2 interaction is an interaction between (at least) two processing streams.

Stage 3 is characterized by the participant's response strategy. This stage addresses whether the participant's expressed judgments of the stimulus dimensions influence one and other. The participant may judge each dimension without consideration of the other dimension, or he or she may conflate the two judgments. Whereas interactions of Stages 1 and 2 are perceptual, Stage 3 interaction is cognitive.

In a version of the processing model above, Ashby (1989) noted associations between (1) the failure of perceptual separability (termed perceptual integrality) and Stage 1 interaction, (2) the failure of perceptual independence (termed perceptual dependence) and Stage 2 interaction, and (3) the failure of decisional separability (termed decisional integrality) and Stage 3 interaction. Although Ashby and Maddox (1994) stated that "stimulus components $\mathrm{X}$ and $\mathrm{Y}$ are perceptually separable if and only if they are processed by separate channels with nonoverlapping tuning curves (i.e., Stage 1 interaction)" (p. 434), Ashby (1989) noted that interactions in Stage 1 or Stage 2 can produce failures of perceptual separability. ${ }^{2}$

\section{Analysis Procedures}

A few specialized analysis procedures have been developed to assess the perceptual and decisional processes in GRT space. ${ }^{3}$ These procedures include Kadlec and Townsend's (1992a, 1992b) macro- and microanalyses, Wickens's (1992) maximum-likelihood model, and Maddox and Ashby's (1996) parameter estimation procedures. Perhaps the most widely implemented of these procedures 


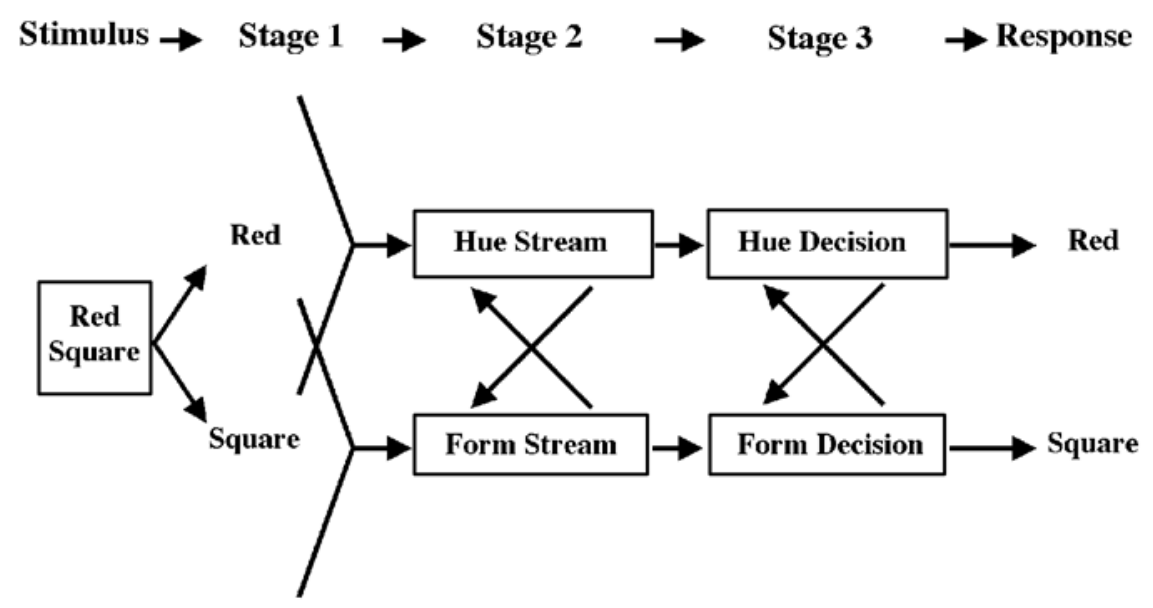

Figure 2. A model of three broad stages of processing during which dimensional interaction may occur.

are Kadlec and Townsend's macro- and microanalyses, which are based on SDT analysis.

Kadlec and Townsend (1992a, 1992b) proposed an experimental and statistical technique based on SDT to assess perceptual separability, perceptual independence, and decisional separability. In its simplest form, they proposed that experimenters conduct a complete identification experiment, using stimuli composed of a factorial combination of two dimensions ( $\mathbf{A}$ and $\mathbf{B})$, with each dimension composed of two values, $i=\{0,1\}$ and $j=\{0,1\}$. In a complete identificationexperiment, participants are briefly presented one of the four stimuli (i.e., $\mathbf{A}_{0} \mathbf{B}_{0}, \mathbf{A}_{1} \mathbf{B}_{0}, \mathbf{A}_{0} \mathbf{B}_{1}$, or $\mathbf{A}_{1} \mathbf{B}_{1}$ ) and are asked to identify which stimulus was presented. Kadlec and Townsend described (1) a macroanalysis, based on univariate marginal distributions, that is used to place at least one axis of each perceptual distribution in GRT perceptual space and (2) a microanalysis, based on conditional $d^{\prime}$ s (i.e., assessing $d^{\prime}$ along Dimension $\mathbf{A}$ conditional on a hit or a miss on Dimension B), that is used to provide some limited information about the variance/covariance matrix of the perceptual distributions. Although Kadlec and Townsend's procedure does not distinguish between perceptual and decisional processes, it can test properties of both perceptual and decisional processes. (For a complete description of macro- and microanalyses, see Kadlec \& Townsend, 1992a, 1992b.)

Several other procedures, based on parameter estimation, have also been proposed. Two such parameter estimation procedures are those of Wickens (1992) and Maddox and Ashby (1996). Wickens's maximum-likelihood model uses a maximum-likelihood procedure to estimate the parameters of each multivariate Gaussian perceptual distribution. The estimation procedure is based on polychoric correlation analysis and assumes decisional separability. Wickens's model does not address the distinction between decisional and perceptual effects. Maddox and Ashby proposed a more extensive parameter estimation approach that addresses the distinction between decisional and perceptual processes by developing and comparing models with different assumptions about these processes (e.g., Maddox, 2001; Maddox \& Ashby, 1996; Maddox \& Bogdanov, 2000). Whereas Maddox and Ashby's procedure provides more precise information about perceptual and decisional processes in multidimensional space than do both Kadlec and Townsend's (1992a, 1992b) macroand microanalyses and Wickens's maximum-likelihood model, it requires large amounts of data to implement (i.e., the degrees of freedom must exceed the number of parameters to be estimated; see Maddox \& Ashby, 1996).

In sum, GRT extends SDT to multidimensional space. Unfortunately, assessment of perceptual and decisional processes in multidimensional space is difficult, and the available procedures to recover information about these processes require specialized statistical programs and skills. Below, I will describe a relatively simple regression model with which to assess the perceptual and decisional processes associated with detecting multidimensional stimuli in GRT space. The regression model can be run on any available statistical analysis program and provides estimates of $\mu$ and $\sum$ for each perceptual distribution assessed.

\section{REGRESSION MODEL}

DeCarlo (1998) has illustrated the advantages of using a generalized linear model to estimate the parameters of unidimensional SDT analysis. Similar to standard SDT analysis, DeCarlo used participants' ratings to identify regions between criteria that intersect underlying logistic or normal distributions. The procedure proposed here uses participants' ratings as direct estimates of the perceptual effects of the stimuli (e.g., Hirsch, Hylton, \& Graham, 1982).

If one has a direct estimate of the perceptual effect of each dimension on every trial of a complete identification experiment, one can estimate the $\mu$ and $\Sigma$ of each perceptual distribution, using a regression model that is theoretically linked to (1) the stages of the processing model 
described above and (2) the perceptual space described in GRT. Ashby (1989) has outlined such a model and the equations that predict its behavior on the basis of both observable and unobserverable variables. I will present a version of Ashby's (1989) equations based solely on observable variables. In the present example, the participant's perceptual effects for each dimension are estimated using a fine ordinal scale of the participant's confidence in the presence or absence of an assigned target value in each dimension (discussion of the assumed qualities of the response scale will be presented below in the Assumptions of the Response Scale section).

The regression model predicts a participant's estimate of the perceptual effect of each stimulus dimension $\left(a_{i}\right.$ and $b_{j}$ ) from (1) the feature value of Dimension $\mathbf{A}\left(\mathbf{A}_{i}\right),(2)$ the feature value of Dimension $\mathbf{B}\left(\mathbf{B}_{j}\right)$, (3) an interaction between the feature values of Dimensions $\mathbf{A}$ and $\mathbf{B}\left(\mathbf{A}_{i} \mathbf{B}_{j}\right)$, and (4) the response on the secondary dimension $\left(a_{i}\right.$ or $b_{j}$-when $a_{i}$ is predicted, Dimension $\mathbf{A}$ is primary and Dimension $\mathbf{B}$ is secondary; when $b_{j}$ is predicted, Dimension $\mathbf{B}$ is primary and Dimension $\mathbf{A}$ is secondary). Thus, for Dimension A,

$a_{i}=\alpha_{a}+\beta_{a 1}\left(\mathbf{A}_{i}\right)+\beta_{a 2}\left(\mathbf{B}_{j}\right)+\beta_{a 3}\left(\mathbf{A}_{i} \mathbf{B}_{j}\right)+\beta_{a 4}\left(b_{j}\right)+e_{a}$,

and similarly for Dimension $\mathbf{B}$,

$b_{j}=\alpha_{b}+\beta_{b 1}\left(\mathbf{B}_{j}\right)+\beta_{b 2}\left(\mathbf{A}_{i}\right)+\beta_{b 3}\left(\mathbf{A}_{i} \mathbf{B}_{j}\right)+\beta_{b 4}\left(a_{i}\right)+e_{b}$.

The response pair $\left(a_{i}, b_{j}\right)$ is assumed to be a direct estimate of the mean of the perceptual distribution associated with each stimulus (i.e., $\mathbf{A}_{0} \mathbf{B}_{0}, \mathbf{A}_{1} \mathbf{B}_{0}, \mathbf{A}_{0} \mathbf{B}_{1}$, and $\mathbf{A}_{1} \mathbf{B}_{1}$ ). $\beta_{._{1}}$ carries the influence of the value of the primary dimension on the participant's response to the primary dimension. $\beta_{._{2}}$ and $\beta_{._{3}}$ carry the influence of the secondary dimension on the participant's response to the primary dimension. Specifically, $\beta_{._{2}}$ carries the additive effect and $\beta_{\cdot 3}$ carries the nonadditive effect of the secondary dimension on the participant's response to the primary dimension. For example, if Dimension A's influence on Dimension B is non-zero and constant across all the stimuli, then $\beta_{b 2} \neq 0$ and $\beta_{b 3}=0$. If Dimension A's influence on Dimension $\mathbf{B}$ is dependent on properties of the stimulus, then $\beta_{b 3} \neq 0$. $\beta_{{ }_{4}}$ carries the influence of the participant's response to the secondary dimension on his or her response to the primary dimension.

The term representing the response on the secondary dimension (i.e., $\beta_{._{4}}$ ) creates a dependency between the two equations. However, because each equation includes identical values of regressors (e.g., $\mathbf{A}_{i}, \mathbf{B}_{j}$, and $\mathbf{A}_{i} \mathbf{B}_{j}$ ), a separate estimation of each equation without the secondary dimension response term (i.e., $\beta .4$ ), together with a correlation of residuals, provides the same information as a simultaneous estimation of the equations with the secondary dimension response terms (Dwivedi \& Srivastava, 1978;
Kmenta, 1986). Therefore, to estimate $a_{i}$ and $b_{j}$, one simply regresses the stimulus variables on each response variable,

$$
a_{i}=\alpha_{a}+\beta_{a 1}\left(\mathbf{A}_{i}\right)+\beta_{a 2}\left(\mathbf{B}_{j}\right)+\beta_{a 3}\left(\mathbf{A}_{i} \mathbf{B}_{j}\right)+e_{a}
$$

and

$$
b_{j}=\alpha_{b}+\beta_{b 1}\left(\mathbf{B}_{j}\right)+\beta_{b 2}\left(\mathbf{A}_{i}\right)+{ }_{b 3}\left(\mathbf{A}_{i} \mathbf{B}_{j}\right)+e_{b},
$$

and assesses the correlation between the residuals of the two equations $\left(\rho_{\mathrm{eAeB}}\right)$.

The interpretation of how the parameters of the regression model relate to the processing model described above and to GRT are summarized in Table 1 . The correlation between the residuals of the Equations $2 \mathrm{a}$ and $2 \mathrm{~b}\left(\rho_{\mathrm{eAeB}}\right)$ indicates whether a dependency exists between the participant's responses to each dimension $\left(a_{i}\right.$ and $\left.b_{j}\right)$. A dependency between a participant's responses to each dimension is assumed to result from either a Stage 2 interaction or a Stage 3 interaction. For example, if the activation of Stream $\mathbf{A}$ influences Stream $\mathbf{B}$ (i.e., a Stage 2 interaction), the noise in Stream A will correlate with the noise in Stream B. If $a_{i}$ and $b_{j}$ are valid estimates of the perceptual effects (an assumption of the regression model), evidence of this correlated noise will be apparent in the participant's responses (i.e., $\rho_{\mathrm{eAeB}} \neq 0$ ). Similarly, if a participant's response on Dimension A influences his or her response on Dimension $\mathbf{B}$ (i.e., a Stage 3 interaction), a participant's responses to each dimension will be correlated (i.e., $\rho_{\mathrm{eAeB}} \neq 0$ ). Thus, although $\rho_{\mathrm{eAeB}}$ indicates whether interstream influence has been exerted, one cannot mathematically determine whether the influence was exerted during processing (Stage 2) or after processing (Stage 3). ${ }^{4}$ In short, if a Stage 2 and/or a Stage 3 interaction occurs, then $\rho_{\mathrm{eAeB}} \neq 0$. The converse is also true; if $\rho_{\mathrm{eAeB}}=0$, then no interaction occurs in Stage 2 or 3 . Because perceptual independence and decisional separability correspond to Stage 2 and Stage 3, respectively, failure of perceptual independence or decisional separability will produce $\rho_{\mathrm{eAeB}} \neq 0$. Likewise, if $\rho_{\mathrm{eAeB}}=$ 0 , one can conclude that perceptual independence and decisional separability hold.

Significant $\beta$ s for the third and/or the fourth terms $\left(\beta_{._{2}}\right.$ and/or $\beta_{.3}$ ) of Equations $2 \mathrm{a}$ and $2 \mathrm{~b}$ indicate mean shift integrality as described by GRT. Mean shift integrality is a form of perceptual integrality in which the perception of the secondary dimension influences the mean perceptual effect of the primary dimension. Because the stimulus variables of the secondary dimension (e.g., $\mathbf{B}_{j}$ and $\mathbf{A}_{i} \mathbf{B}_{j}$ ) contribute to perceptual integrality (i.e., $\beta_{._{2}} \neq 0$ and/or $\left.\beta_{\cdot 3} \neq 0\right)$ and these same stimulus variables influence the dependent term (e.g., $\beta .4$ ), both the original stimulus variables and the dependent term can produce perceptual integrality (recall that when implementing Equations $2 a$ and $2 \mathrm{~b}$, the influence of the dependent term is expressed in $\rho_{\text {eAeB }}$. Therefore, Stage 1, 2, and 3 interactions may all be 
Table 1

A Summary of the Relation Between the Parameters of the Regression Model, the Processing Model, and General Recognition Theory (GRT)

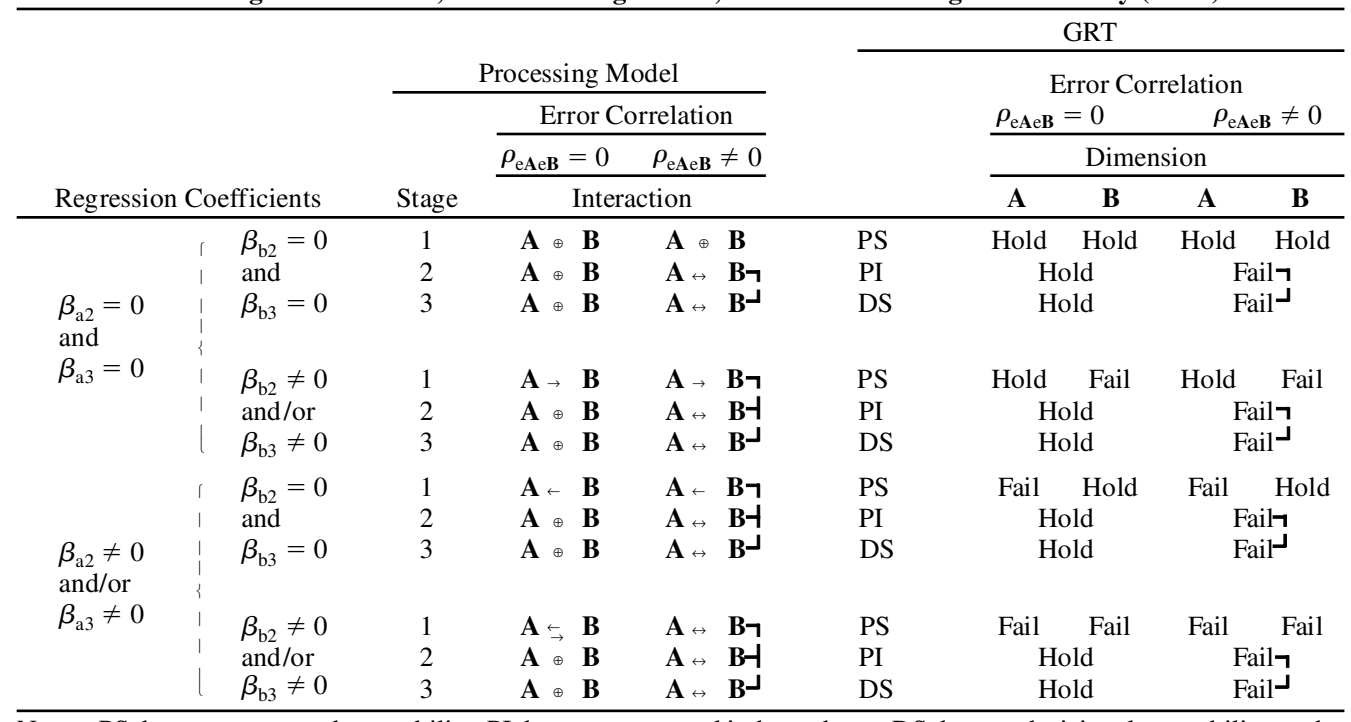

Note-PS denotes perceptual separability; PI denotes perceptual independence; DS denotes decisional separability; $\oplus$ denotes no interaction; $\rightarrow$ and $\leftarrow$ denote that an interaction is present and indicate the direction of influence (e.g., $\mathbf{A} \rightarrow \mathbf{B}$ indicates that Dimension A influences the perception (Stage 1 or 2) or the decision (Stage 3) processes of Dimension $\mathbf{B}$ ); $\leftrightarrow$ denotes that an interaction is present but the direction of the influence is indeterminate; brackets ( 1 抽) indicate that at least one of the interactions connected with the brackets is present.

a source of perceptual integrality. To intuitively understand how the dependent term can produce perceptual integrality, suppose that one's perception of form $\left(\psi_{\text {form }}\right)$ is a function of the two following stimulus variables: the form dimension $\left(\mathbf{F}_{i}\right)$ and an interaction between the form and the hue dimensions $\left(\mathbf{F}_{i} \mathbf{H}_{j}\right)$. Further suppose that one's perception of hue $\left(\psi_{\text {hue }}\right)$ is influenced by the hue dimension, $\mathbf{H}_{j}$, and $\psi_{\text {form }}$ (i.e., a Stage 2 interaction). In this instance, the participant's hue response will be influenced by $\mathbf{H}_{j}$ directly and by $\mathbf{F}_{i}$ and $\mathbf{F}_{i} \mathbf{H}_{j}$ indirectly (through $\psi_{\text {form }}$ ). Thus, the hue response will appear to be influenced by all three stimulus variables (e.g., $\mathbf{F}_{i}, \mathbf{H}_{j}$, and $\mathbf{F}_{i} \mathbf{H}_{j}$ ) and have correlated residuals with the form description. A similar interaction at Stage 3 produces the same result. Therefore, perceptual integrality (as defined by GRT) uniquely specifies a Stage 1 interaction only when $\rho_{\mathrm{eAeB}}=0$. If a perceptual integrality exists and $\rho_{\mathrm{eAeB}} \neq 0$, one cannot mathematically determine which of the three stages produced the perceptual integrality.

\section{Perceptual Versus Decisional Effects}

The relation between the parameters of the regression model and GRT illustrates the importance of establishing decisional separability. That is, because decisional integrality (Stage 3 interaction) can produce patterns of data that mimic both perceptual integrality and perceptual dependence, if decisional separability fails one cannot determine whether any or all effects present are the result of perceptual or cognitive processes. Fortunately, the regression model provides some limited information about decisional separability. That is, failure of decisional separability will, by definition, result in dependence between the participant's responses to each dimension. Therefore, if $\rho_{\mathrm{eAeB}}=0$, decisional separability must hold. Nevertheless, if $\rho_{\mathrm{eAeB}} \neq 0$, the regression model cannot mathematically distinguish decisional integrality from perceptual dependence.

The regression model is not alone in its difficulty in differentiating a participant's decision strategy from his or her perception in multidimensionalSDT space. When one generalizes SDT to a multidimensional space, it becomes exceedingly difficult to determine the multidimensional response bias (i.e., decisional separability). Kadlec and Townsend (1992b) proposed that micro- and macroanalyses can provide some clues concerning the participant's twodimensional decision strategy if one (1) assumes linear decision bounds and (2) has some knowledge of the placement and shape of the perceptual distributions. Because one must have some information concerning the perceptual space to determine the decision space (Kadlec \& Townsend, 1992b), the information about two-dimensional response bias that one can glean from micro- and macroanalyses is limited (see Kadlec \& Townsend, 1992a).

In the absence of convenient statistical techniques for differentiating a participant's decision strategy from his or her perception in multidimensional SDT space, researchers have turned to logical arguments and/or a priori assumptions. For example, often researchers assume decisional separability and, therefore, attribute interactions to Stages 1 and/or 2 (e.g., Olzak, 1986; Wickens, 1992; Wickens \& Olzak, 1992). Others have assumed that participants use a single response strategy during experimental sessions. 
Therefore, if perceptual and decisional separability holds for one dimension, researchers assume that any interactions present in other dimensions are not due to a Stage 3 interaction (Ashby, 1989). Finally, researchers implement experimental techniques to encourage decisional separability. For example, Maddox and Bogdanov (2000) used a response-terminated matching task to assess a participant's perceptual space, because they assumed that such a task would minimize decisional integrality. The authors later confirmed their model with a separate categorization task.

In light of the fact that the regression model is limited in its ability to distinguish perceptual from decisional processes, it is recommended that researchers implement experimental controls to encourage decisional separability and, if $\rho_{\mathrm{eAeB}} \neq 0$, assess whether the pattern of interactions can be more parsimoniously explained by decisional or perceptual processes (e.g., Ashby, 1989). The following experimental controls may encourage decisional separability: (1) requiring participants to rate each dimension separately, (2) randomizing trial type and order of responses, and (3) not providing corrective feedback. Specifically, Ashby and Maddox (1994) suggested that a naive participant may identify dimensions separately if instructed to do so, even if doing so does not result in optimal performance. Furthermore, by randomizing trial type and order of responses, participants are prevented from devising stimulus-specific attentional and response strategies, because they cannot anticipate which stimulus will be presented and which dimension will be requested first. Finally, because corrective feedback may prompt even a naive participant to adopt an optimal decision strategy (even if the optimal response strategy is not decisionally separable), corrective feedback should be avoided.

\section{Assumptions of the Response Scale}

Perhaps the most formidable obstacle to implementing the regression model is the choice of the response scale used to estimate the perceptual effects of each dimension. Most response scales require participants to assign numbers to the perceived magnitudes of stimuli (e.g., magnitude estimation, category rating, etc.). Although the regression model does not constrain the form of this scale, it does assume that a participant's estimates are valid. The issues associated with the validity of numerical estimates of perceptual experiences have been discussed extensively elsewhere (e.g., Gescheider, 1988; Marks \& Algom, 1998; Stevens, 1986). Generally speaking, the validity of subjective reports of internal experience cannot be unequivocally established and, therefore, must be assumed.

Numerical estimates of perceptual effects have been used successfully to map unidimensional space (Braida \& Durlach, 1972; Cohen \& Lecci, 2001; Durlach \& Braida, 1969). Specifically, Durlach and Braida presented a formal decision model that links a participant's numerical descriptions of the perceptual effect of a stimulus to the hypothesized underlying distributions of unidimensional SDT. Braida and Durlach (1972) provided empirical evidence that numerical descriptions (i.e., magnitude estimates, absolute estimates, and category ratings) and unidimen- sional SDT analysis may afford equivalent information. Recently, Cohen and Lecci generalized this model to assess the perceptual effect of specific dimensions of a stimulus. If the practical application of these findings can be generalized to a multidimensional perceptual space, then one may use these measures (i.e., magnitude estimates, absolute estimates, and category ratings) to map GRT perceptual space, using the regression model.

Researchers assessing dimensional interaction in multidimensional space are interested in the relative effects of one stimulus dimension on the perception of the other stimulus dimension. Such an assessment requires that the participant apply the response scale to the perceptual effects according to a rule (other than randomness; Stevens, 1986). Such a mapping ensures that the participant's estimates are ordinally related to the actual perceptual effects but does not ensure equal intervals between successive numbers. Finally, the mapping rule need not be the same for each dimension assessed. Therefore, to assess dimensional interaction, using the regression model, the participant's estimates must be at least ordinally related to his or her perceptual experiences, and decisional separability must hold.

For example, one may assess the effects of Dimension A on the perception of Dimension B (i.e., perceptual separability) by comparing, along Dimension $\mathbf{B}$, (1) the means and variances of $\mathbf{A}_{0} \mathbf{B}_{0}$ and $\mathbf{A}_{1} \mathbf{B}_{0}$ and (2) the means and variances of $\mathbf{A}_{0} \mathbf{B}_{1}$ and $\mathbf{A}_{1} \mathbf{B}_{1}$. If perceptual separability holds for Dimension $\mathbf{B}, \mathbf{A}_{0} \mathbf{B}_{0}$ should be identical to $\mathbf{A}_{1} \mathbf{B}_{0}$, and $\mathbf{A}_{0} \mathbf{B}_{1}$ should be identical to $\mathbf{A}_{1} \mathbf{B}_{1}$, along Dimension $\mathbf{B}$. Furthermore, if decisional separability holds for Dimension B, participants' criteria will be perpendicular to the axis representing Dimension $\mathbf{B}$. If both perceptual and decisional separability holds, participants' ratings will intersect $\mathbf{A}_{0} \mathbf{B}_{0}$ in exactly the same places that they intersect $\mathbf{A}_{1} \mathbf{B}_{0}$, and participants' ratings will intersect $\mathbf{A}_{0} \mathbf{B}_{1}$ in exactly the same places that they intersect $\mathbf{A}_{1} \mathbf{B}_{1}$, regardless of the ordinal scale that the participantadopts. Therefore, if the participant's perceptual and decisional processes associated with Dimension B are unaffected by Dimension A (i.e., perceptual and decisional separability), the means and variances of the participant's ordinal estimates of Dimension $\mathbf{B}$ will not differ for $\mathbf{A}_{0} \mathbf{B}_{0}$ and $\mathbf{A}_{1} \mathbf{B}_{0}$ or $\mathbf{A}_{0} \mathbf{B}_{1}$ and $\mathbf{A}_{1} \mathbf{B}_{1}$. Similarly, the assessment of perceptual separability is not affected by unidimensional bias. That is, if decisional and perceptual separability holds and a participant has a liberal bias when estimating the perceptual effects of Dimension B, this bias will have equal effects on the participant's estimates of Dimension $\mathbf{B}$ for all stimuli, regardless of the level of Dimension A. If either decisional or perceptual separability fails, the estimates of the mean and variance of the relevant perceptual distributions will be unequal, again, regardless of the ordinal scale that the participant adopts and any unidimensional response bias.

Ordinal estimates also provide information about perceptualindependence. Ashby (1988) has shown that the interrating correlation is related to the correlation between the underlying perceptual distributions. However, Ashby (1988) also has shown that unidimensional response bias and the participant's choice of an ordinal scale may result in an un- 
derestimation of that relation. Nevertheless, the presence or absence of a relation can be assessed with sufficient power.

Although ordinal measures of perceptual effects can be used to assess the presence or absence of dimensional interaction, they are limited in two ways. First, the participant's implementation of the ordinal scale may result in reduced statistical power. Specifically, the greater the inequality of intervals between successive numbers, the greater the reduction in statistical power. Second, the ordinal measure does not provide information about the $a b$ solute magnitudes of effects. To draw conclusions about absolute magnitudes, one must assume that the participant's estimates carry more than ordinal information.

The appropriateness of the regression model is also complicated by the statistical assumption that the dependent variable contains at least interval information. Conover and Iman (1981) have shown, however, that applying a standard parametric procedure (such as a multiple regression) to ranked scores (which are equivalent to ratings data) results in a powerful test that makes no distributional or interval assumptions. Therefore, these tests are appropriate for use with an ordinal measure of perceptual effects if one draws conclusions only about relative magnitudes.

In sum, the regression model makes several assumptions about the information contained in a participant's numerical responses. If these assumptions hold (or are common across analytic procedures), the regression model should produce the same conclusions as more established analytic procedures. Thus, to validate the regression model, I (1) conducted a concurrent ratings experiment in which the perceptual space associated with hue and form was assessed, using isoluminant stimuli, (2) mapped the perceptual space, using both Kadlec and Townsend's (1992a, 1992b) macro- and microanalyses and the regression model, and (3) compared the results of the two analyses. Kadlec and Townsend's analytic procedure was chosen as a comparison because it is founded on SDT analysis and the validity of SDT analysis has been well studied (e.g., Green \& Swets, 1966; Macmillan \& Creelman, 1991).

\section{EXPERIMENT}

The present experiment is an extension of Cohen (1997). Cohen reported a visual detection experiment that provided evidence that color and form are not detected independently in early vision. The present experiment extended Cohen by using isoluminant stimuli and obtaining separate estimates of the perceptual effect of each perceptual dimension.

\section{Method}

\section{Participants}

Sixteen participants from the general psychology subject pool volunteered to participate in three sessions over 3 days.

\section{Apparatus and Stimuli}

All the stimuli were presented on a 15 -in. color monitor with a 60 $\mathrm{Hz}$ refresh rate, controlled by a Pentium microcomputer using a DOS operating system. The resolution of the monitor was $800 \times 600$.

A set of elements was constructed that was a factorial combination of two hues and two forms. Each element subtended $1^{\circ}$ of visual angle. The two hues were isoluminant shades of purple and blue. ${ }^{5}$ Isoluminance was achieved for each participant by using a flicker fusion task prior to the experiment. In the flicker fusion task, each participant was presented a square patch of color, subtending $1^{\circ}$ of visual angle, that alternated between the two colors at the speed of the refresh rate (16.7 msec; using palette animation). Initially, there was a perceptual flicker when the shades alternated. The participant was instructed to adjust the luminance of one of the colors until he or she could not see the patch flicker. The participant adjusted the luminance by using the 4 and 6 keys on the keypad on the keyboard. The 4 key increased the luminance and the 6 key decreased the luminance of the color. When the participant no longer perceived the flicker, he or she hit the Enter key. The resulting values were used as the two hues in the visual detection task described below.

The two levels of the form dimension were a square and an octagon. The length of the oblique sides of the octagon was adjusted in a pilot experiment so that the participants were equally sensitive to variation in the hue and the form dimensions. Whereas the luminance of the hue dimension was adjusted for each participant, the shapes of the stimuli were constant across participants.

For each participant, one feature value from each feature dimension was chosen as a target. The target values were counterbalanced between participants. The target value is noted with a subscript of 1 , and the nontarget value is noted with a subscript of 0 . Thus there were four types of elements: (1) a nontarget element, $\mathbf{F}_{0} \mathbf{H}_{0}$, (2) a form target element, $\mathbf{F}_{1} \mathbf{H}_{0}$, (3) a hue target element, $\mathbf{F}_{0} \mathbf{H}_{1}$, and (4) a form and hue target element, $\mathbf{F}_{1} \mathbf{H}_{1}$.

Each trial contained four elements arranged in a square around the center of the computer monitor. This matrix of four elements subtended $5^{\circ}$ of visual angle on a side. There were five trial types (see Figure 3): (1) a no-target trial composed of four $\mathbf{F}_{0} \mathbf{H}_{0}$ elements, (2) a form target trial composed of three $\mathbf{F}_{0} \mathbf{H}_{0}$ elements and one $\mathbf{F}_{1} \mathbf{H}_{0}$, (3) a hue target trial composed of three $\mathbf{F}_{0} \mathbf{H}_{0}$ elements and one $\mathbf{F}_{0} \mathbf{H}_{1}$, (4) a coincident target trial composed of three $\mathbf{F}_{0} \mathbf{H}_{0}$ elements and one $\mathbf{F}_{1} \mathbf{H}_{1}$, and (5) a disparate target trial composed of two $\mathbf{F}_{0} \mathbf{H}_{0}$ elements, one $\mathbf{F}_{1} \mathbf{H}_{0}$, and one $\mathbf{F}_{0} \mathbf{H}_{1}$.

\section{Target Hue: Black \\ Target Form: Square}

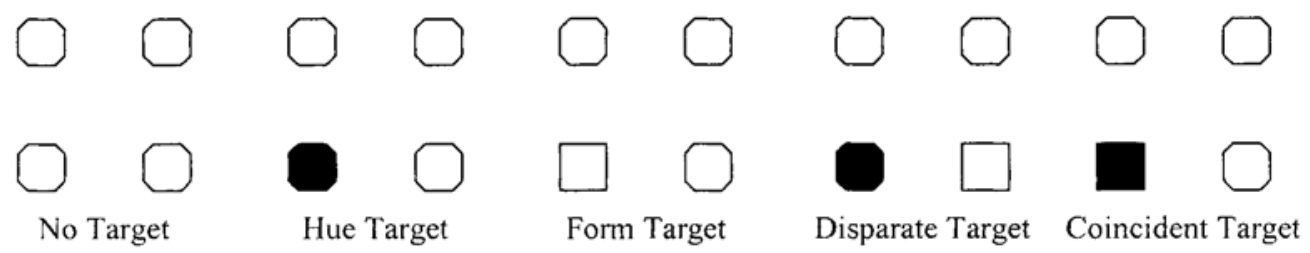

Figure 3. Examples of the five trial types in the present experiment. 
On those trials in which at least one target element was present, the target element was presented in a randomly chosen spatial position. The target elements were presented in random locations to discourage the participants from focusing their attention on a restricted portion of the display. Each positive trial type (trials that contained target elements) was presented with a probability of .125 , and the negative trials (trials that contained no target elements) were presented with a probability of .5. Randomly presenting the trial types discouraged the participants from relying on a trial-specific strategy. The display time was manipulated between participants. Each participant was presented displays for either 33 or $83 \mathrm{msec}$.

Because Braida and Durlach (1972) had shown showed that magnitude estimates and category ratings provide equivalent information in a detection task, an 18-point ordinal response scale was used in the present experiment. Eighteen categories were chosen because participants generally use between 10 and 20 numbers in a magnitude estimation task (Luce \& Krumhansl, 1988). Furthermore, when a set of restricted responses is used, the results are essentially unchanged if that set is sufficiently large (Durlach \& Braida, 1969). To facilitate the participant's use of the entire scale, the response screens were constructed to be a cross between a visual and a semantic scale. Specifically, the numbers 1 through 18 were listed horizontally across the screen and the participant moved a box across the numbers to indicate his or her response. Thus, both the number and the position of the box were indicators of magnitude.

There was a response screen for each dimension assessed. One response screen asked the participant to rate his or her confidence that the target form feature value was present in the display. At the top of this response screen, the following sentence was written: "Please rate your confidence that the target FORM was present." Underneath this sentence, the word form was written in large letters centered horizontally on the screen. Below the word form, the numbers 1-18 were listed horizontally across the computer monitor. Over the number 1, the words very sure no target present were written. Over the number 18, the words very sure target present were written. The number 1 was framed by a box. The participant manipulated the position of the box by pressing the 4 and 6 keys on the computer keypad. The 4 key moved the box to the left; the 6 key moved the box to the right. When the box was moved to left past the rating of 1 (or to the right past the rating of 18), it wrapped around the rating scale to the number 18 (or 1). When the participant positioned the box over his or her intended rating, he or she hit the Enter key to finalize his or her choice. The second response screen asked the participant to rate his or her confidence that the hue target feature value was present. This screen was identical to the form response screen, with the exception that the word form was replaced by the word color throughout. ${ }^{6}$ On every trial the participant responded to both response screens. The order in which the response screens were presented was randomly determined on each trial.

\section{Procedure}

Each participant was tested individually in a small dark room. The participant was given both verbal and written instructions. The instructions indicated which target feature values the participant was to identify. The participant was shown those features, as well as the nontarget feature values. The participant was shown self-timed examples of each trial type. When the participant indicated understanding of the task, the experiment began.

Each trial began with a 500-msec blank screen, followed by the trial matrix and by a mask of yellow stars. The mask remained visible for $1 \mathrm{sec}$. Following the mask, the two response screens were presented in random order. There was no feedback.

Each participant participated in three sessions over the course of 1 week. Each session consisted of 40 practice trials, followed by 480 experimental trials per session. The practice trials contained 5 trials of each of the four target trial types and 20 nontarget trials. The experimental trials contained 60 trials of each of the four target trial types and 240 nontarget trials. The trial types were presented randomly within and across sessions. The participant was allowed one self-timed break after 240 trials.

\section{Results}

Below are the results of the micro- and macroanalyses, followed by those of the regression analysis. Each analysis was first calculated on the individual participant's data and then concatenated across participants using a summed$z$ meta-analysis (for a detailed description of the analysis, see Cohen, 1997; Rosenthal \& Rubin, 1986; Strube, 1985; Strube \& Miller, 1986). The summed-z meta-analysis was appropriate because the degrees of freedom associated with each $t$ test were large enough for the $t$ distribution to be a good approximation of a Gaussian. The meta-analysis was particularly well suited to the present data because it emphasized individual effects while simultaneously providing an overall summary of the data. ${ }^{7}$ Separate analyses were performed for each condition $\times$ display time (33 vs. $83 \mathrm{msec}$ ). All reported $p$ values indicate significance as calculated by the meta-analysis, and because multiple tests per condition were calculated, $\alpha=.01$.

\section{Use of the Rating Scale}

The precision of the regression analysis is dependent in part on the participant's ability to apply the rating scale. If the participant uses only a portion of the scale, the power to detect perceptual integrality and perceptual dependence is reduced. In general, although most of the participants used the entire response scale, they tended to gravitate toward the ratings on the high and low ends of the scale. Specifically, the participants' average form rating was 11.49 $(S D=7.36)$, and the average color rating was 8.46 $(S D=8.09)$. When assessing form, only 2 participants did not use the entire 18 response criteria (those 2 participants used 14 and 16 response criteria). When assessing hue, 9 participants did not use the entire 18 response criteria, with 8 of those 9 participants using 15 or more response criteria (the 9th participant used 8 response criteria). When a target was presented, the participants used Ratings 13 and greater $89 \%$ of the time for form responses $(M=16.27, S D=4.55)$ and $95 \%$ of the time for hue responses $(M=17.16, S D=3.48)$. When a target was not presented, the participants used Ratings 1-6 49\% of the time for form responses $(M=8.62, S D=7.24)$ and $85 \%$ of the time for hue responses $(M=6.00, S D=5.00)$.

\section{Macro- and Microanalyses}

Because the participants tended to gravitate toward the ratings on the high end of the scale when the target was presented, Ratings 13-17 (inclusive) were used as criteria when the micro- and macroanalyses were calculated. As would be predicted by decisional separability, the results were stable across criteria. Therefore, the marginal $d^{\prime}$ and marginal response invariance (MRI) results are reported only for Criterion 15 and are displayed in Tables 2 and 3.

Marginal $\boldsymbol{d}^{\prime}$. The marginal $d^{\prime}$ analysis assesses whether the average perceptual effect of one dimension depends 
Table 2

A Summary of the Results of the Macroanalyses as They Relate to Perceptual Separability for Display Times of 33 and $83 \mathrm{msec}$

\begin{tabular}{|c|c|c|c|c|c|c|c|}
\hline \multirow[b]{2}{*}{ Dimension } & \multirow[b]{2}{*}{ Time (msec) } & \multicolumn{4}{|c|}{ Marginal $d^{\prime}$} & & \\
\hline & & $\begin{array}{c}\text { Coincident } \\
\left(\mathbf{F}_{1} \mathbf{H}_{1}\right)\end{array}$ & $p$ & $\begin{array}{c}\text { Disparate } \\
\left(\mathbf{F}_{1} \mathbf{H}_{1}\right)\end{array}$ & $p$ & & \\
\hline \multirow[t]{2}{*}{ Form } & 33 & $\begin{array}{l}d^{\prime}{ }_{\mathrm{FH} 0}=2.31 \\
d_{\mathrm{FH} 1}^{\prime}=2.23\end{array}$ & n.s. & $\begin{array}{l}d_{\mathrm{FH} 0}^{\prime}=2.31 \\
d_{\mathrm{FH} 1}^{\prime}=1.75\end{array}$ & $<.001$ & & \\
\hline & 83 & $\begin{array}{l}d^{d^{\prime}{ }^{\prime} 0}=1.86 \\
d^{\prime}{ }_{\mathrm{FH} 1}=2.32\end{array}$ & $<.001$ & $\begin{array}{l}d^{\prime}{ }_{\text {FH } 0}=1.86 \\
d^{\prime}{ }_{\text {FH1 }}=1.22\end{array}$ & $<.001$ & & \\
\hline \multirow[t]{4}{*}{ Hue } & 33 & $\begin{aligned} d_{\mathrm{FOH}}^{\prime} & =3.28 \\
d_{\mathrm{FlH}}^{\prime} & =3.34\end{aligned}$ & n.s. & $\begin{array}{l}d^{\prime}{ }_{\mathrm{FOH}}=3.28 \\
d^{\prime}=3.32\end{array}$ & n.s. & & \\
\hline & 83 & $\begin{array}{l}d_{\mathrm{FOH}}^{\prime}=3.38 \\
d_{\mathrm{F} 1 \mathrm{H}}^{\prime}=3.68\end{array}$ & $<.01$ & $\begin{array}{l}d_{\mathrm{FOH}}^{\prime}=3.38 \\
d_{\mathrm{F} 1 \mathrm{H}}^{\prime}=3.40\end{array}$ & n.s. & & \\
\hline & & \multicolumn{6}{|c|}{ MRI } \\
\hline & & $\begin{array}{c}\text { Coincident } \\
\left(\mathbf{F}_{1} \mathbf{H}_{1}\right)\end{array}$ & $p$ & $\begin{array}{c}\text { Disparate } \\
\left(\mathbf{F}_{1} \mathbf{H}_{1}\right)\end{array}$ & $p$ & $\begin{array}{c}\text { No Target } \\
\left(\mathbf{F}_{0} \mathbf{H}_{0}\right)\end{array}$ & $p$ \\
\hline \multirow[t]{2}{*}{ Form } & 33 & $\begin{array}{l}p\left(f_{l} \mid \mathbf{F}_{1} \mathbf{H}_{0}\right)=.86 \\
p\left(f_{l} \mid \mathbf{F}_{1} \mathbf{H}_{1}\right)=.88\end{array}$ & n.s. & $\begin{array}{l}p\left(f_{l} \mid \mathbf{F}_{1} \mathbf{H}_{0}\right)=.86 \\
p\left(f_{l} \mid \mathbf{F}_{1} \mathbf{H}_{1}\right)=.81\end{array}$ & $<.001$ & $\begin{array}{l}p\left(f_{0} \mid \mathbf{F}_{0} \mathbf{H}_{0}\right)=.73 \\
p\left(f_{0} \mid \mathbf{F}_{0} \mathbf{H}_{1}\right)=.69\end{array}$ & $<.001$ \\
\hline & 83 & $\begin{array}{l}p\left(f_{l} \mid \mathbf{F}_{1} \mathbf{H}_{0}\right)=.88 \\
p\left(f_{l} \mid \mathbf{F}_{1} \mathbf{H}_{1}\right)=.96\end{array}$ & $<.001$ & $\begin{array}{l}p\left(f_{l} \mid \mathbf{F}_{1} \mathbf{H}_{0}\right)=.88 \\
p\left(f_{l} \mid \mathbf{F}_{1} \mathbf{H}_{1}\right)=.74\end{array}$ & $<.001$ & $\begin{array}{l}p\left(f_{0} \mid \mathbf{F}_{0} \mathbf{H}_{0}\right)=.67 \\
p\left(f_{0} \mid \mathbf{F}_{0} \mathbf{H}_{1}\right)=.64\end{array}$ & $<.001$ \\
\hline \multirow[t]{2}{*}{ Hue } & 33 & $\begin{array}{l}p\left(h_{l} \mid \mathbf{F}_{0} \mathbf{H}_{1}\right)=.93 \\
p\left(h_{l} \mid \mathbf{F}_{1} \mathbf{H}_{1}\right)=.94\end{array}$ & n.s. & $\begin{array}{l}p\left(h_{l} \mid \mathbf{F}_{0} \mathbf{H}_{1}\right)=.93 \\
p\left(h_{l} \mid \mathbf{F}_{1} \mathbf{H}_{1}\right)=.93\end{array}$ & n.s. & $\begin{array}{l}p\left(h_{0} \mid \mathbf{F}_{0} \mathbf{H}_{0}\right)=.91 \\
p\left(h_{0} \mid \mathbf{F}_{1} \mathbf{H}_{0}\right)=.90\end{array}$ & n.s. \\
\hline & 83 & $\begin{array}{l}p\left(h_{l} \mid \mathbf{F}_{0} \mathbf{H}_{1}\right)=.94 \\
p\left(h_{l} \mid \mathbf{F}_{1} \mathbf{H}_{1}\right)=.98\end{array}$ & $<.001$ & $\begin{array}{l}p\left(h_{l} \mid \mathbf{F}_{0} \mathbf{H}_{1}\right)=.94 \\
p\left(h_{l} \mid \mathbf{F}_{1} \mathbf{H}_{1}\right)=.95\end{array}$ & n.s. & $\begin{array}{l}p\left(h_{0} \mid \mathbf{F}_{0} \mathbf{H}_{0}\right)=.94 \\
p\left(h_{0} \mid \mathbf{F}_{1} \mathbf{H}_{0}\right)=.93\end{array}$ & n.s. \\
\hline
\end{tabular}

Note-Perceptual separability is assessed separately for coincident and disparate targets. Perceptual separability fails for a dimension (form or hue) if the compared measures differ $(p<.01)$. Perceptual separability fails for both coincident and disparate conditions within a dimension if the MRI no-target measures differ.

on the level of the other dimension. Specifically, marginal $d^{\prime}$ analysis assesses the relation between the means of the perceptual distributions. For marginal $d$ 's to be equal, the following must be true:

$$
d_{\dot{\mathbf{A B}} \mathbf{B}_{0}}=d_{\mathbf{A B}_{1}} .
$$

When the participantsidentified the stimulus form, marginal $d^{\prime}$ s were equal only in the 33-msec coincident condition (first two rows of Table 2). For display times of 33 and $83 \mathrm{msec}$, the participants were more sensitive to the form target feature value in the form-only condition than in the disparate condition. Thus, the presence of a hue target feature value in a location spatially different from that of the form target feature value inhibited detection of the form target feature value. For a display time of $83 \mathrm{msec}$, the participants were less sensitive to a form target feature value in the form-only condition than in the coincident condition. Thus, the presence of a hue target feature value in the same spatial location as the form target feature value enhanced detection of the form target feature value.

When the participants identified the stimulus hue, marginal $d^{\prime}$ s were equal in the disparate condition for both display times and in the 33-msec coincident condition (third and fourth rows of Table 2). For a display time of $83 \mathrm{msec}$, the participants were less sensitive to a hue target feature value in the hue-only condition than in the coincident con-

Table 3

A Summary of the Results of the Microanalyses as They Relate to Perceptual Independence for Display Times of 33 and $83 \mathrm{msec}$

\begin{tabular}{|c|c|c|c|c|c|c|c|c|}
\hline \multirow{2}{*}{$\begin{array}{c}\text { Time } \\
(\mathrm{msec})\end{array}$} & & & \multicolumn{3}{|c|}{ Sampling Independence } & \multicolumn{3}{|c|}{ Tetrachoric Correlation } \\
\hline & \multicolumn{2}{|c|}{ Stimulus } & $p\left(f_{1} h_{1} \mid \mathbf{F}_{i} \mathbf{H}_{j}\right)$ & Independence & $p$ & $\rho$ & $S E$ & $p$ \\
\hline \multirow[t]{5}{*}{33} & no target & $\left(\mathbf{F}_{0} \mathbf{H}_{0}\right)$ & .048 & .03 & $<.001$ & .48 & .07 & $<.001$ \\
\hline & form & $\left(\mathbf{F}_{1} \mathbf{H}_{0}\right)$ & .072 & .073 & n.s. & -.15 & .17 & n.s. \\
\hline & hue & $\left(\mathbf{F}_{0} \mathbf{H}_{1}\right)$ & .293 & .282 & n.s. & .30 & .17 & $<.001$ \\
\hline & disparate & $\left(\mathbf{F}_{1} \mathbf{H}_{1}\right)$ & .779 & .764 & n.s. & .62 & .28 & $<.001$ \\
\hline & coincident & $\left(\mathbf{F}_{1} \mathbf{H}_{1}\right)$ & .858 & .838 & n.s. & .77 & .35 & $<.001$ \\
\hline \multirow[t]{5}{*}{83} & no target & $\left(\mathbf{F}_{0} \mathbf{H}_{0}\right)$ & .037 & .025 & $<.001$ & .24 & .08 & $<.001$ \\
\hline & form & $\left(\mathbf{F}_{1} \mathbf{H}_{0}\right)$ & .059 & .063 & n.s. & -.36 & .19 & $<.001$ \\
\hline & hue & $\left(\mathbf{F}_{0} \mathbf{H}_{1}\right)$ & .347 & .345 & n.s. & .16 & .18 & n.s. \\
\hline & disparate & $\left(\mathbf{F}_{1} \mathbf{H}_{1}\right)$ & .712 & .709 & n.s. & .42 & .25 & $<.001$ \\
\hline & coincident & $\left(\mathbf{F}_{1} \mathbf{H}_{1}\right)$ & .942 & .937 & n.s. & .72 & .37 & $<.001$ \\
\hline
\end{tabular}

Note-Perceptual independence is assessed separately for each stimulus. Perceptual independence fails if $p<.01$. 


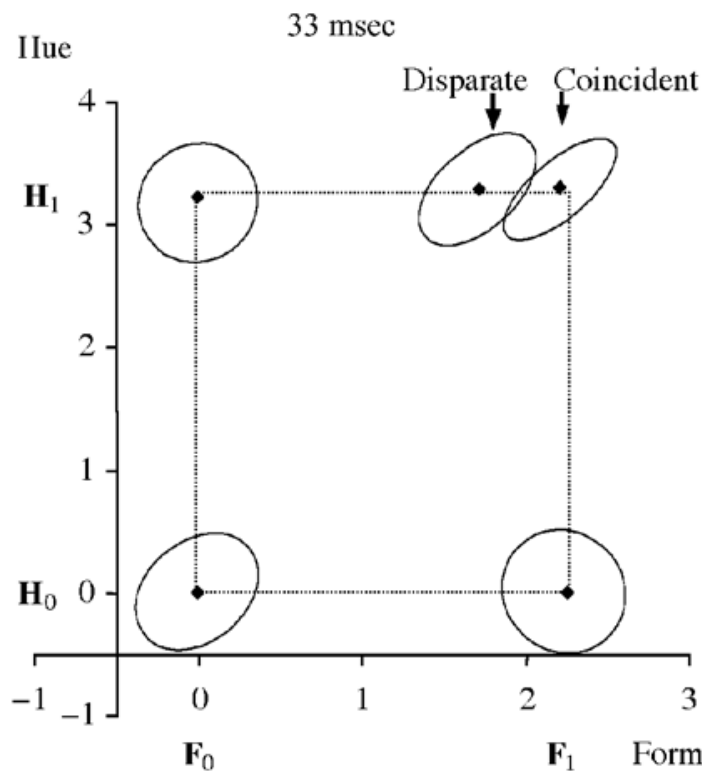

$83 \mathrm{msec}$

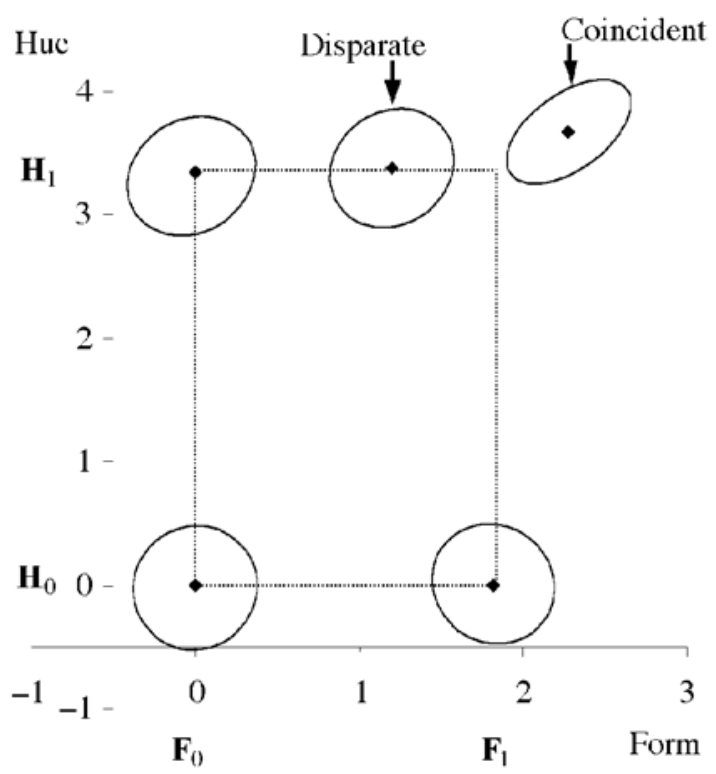

Figure 4. Placement and shapes of the five perceptual distributions associated with the five trial types used in two-dimensional general recognition theory space, based on marginal $d$ 's. The dotted rectangle indicates the predicted position of the bivariate means of the perceptual distributions if hue and form are perceptually separable. The solid ellipses represent equal likelihood contours of the perceptual distributions derived from the results of the tetrachoric correlation analysis, assuming equal variance.

dition. Thus, at $83 \mathrm{msec}$, the presence of a form target feature value in the same spatial location as the hue target feature value enhanced detection of the hue target feature value.

Figure 4 shows the mean placement of the perceptual distributions, using a technique described in Kadlec and Hicks (1998). Specifically, the mean of the $\mathbf{F}_{0} \mathbf{H}_{0}$ percep- tual distributionis placed at $(0,0)$. The remaining perceptual distributions were placed at the distance specified by the relevant marginal $d^{\prime}$ from the $\mathbf{F}_{0} \mathbf{H}_{0}$ perceptual distribution. For the two stimuli that lacked a marginal $d^{\prime}$ on one axis (i.e., $\mathbf{F}_{0} \mathbf{H}_{1}, \mathbf{F}_{1} \mathbf{H}_{0}$ ), the distance on that axis was assumed to be zero. If perceptual separability holds for a dimension, the bivariate means of the perceptual distributions will fall on the dotted lines perpendicular to that dimension's axis. Because of the method used to construct Figure 4, this condition is free to fail only for the dual target distributions. Perceptual independence is represented in Figure 4 by equal likelihood contours based on the tetrachoric correlations, assuming bivariate normal distributions and equal variances. Perceptual independence holds only if the major and minor axes of the ellipse representing equal likelihood contours are parallel to the hue and form axes.

Marginal response invariance. The MRI analysis assesses whether the probability of correctly recognizing one dimension depends on the level of the other dimension. Whereas marginal $d^{\prime}$ provides information about the placement of the perceptual distributions, MRI will fail if (1) $\mu_{\mathbf{A}_{i} \mathbf{B}_{0}} \neq \mu_{\mathbf{A}_{i} \mathbf{B}_{1}}$ or (2) $\sigma_{\mathbf{A}_{i} \mathbf{B}_{0}}^{2} \neq \sigma_{\mathbf{A}_{i} \mathbf{B}_{1}}^{2}$. For MRI to hold, the following must be true (Ashby \& Townsend, 1986):

$$
\begin{aligned}
& p\left(a_{i} b_{0} \mid \mathbf{A}_{i} \mathbf{B}_{0}\right)+p\left(a_{i} b_{1} \mid \mathbf{A}_{i} \mathbf{B}_{0}\right) \\
& \quad=p\left(a_{i} b_{0} \mid \mathbf{A}_{i} \mathbf{B}_{1}\right)+p\left(a_{i} b_{1} \mid \mathbf{A}_{i} \mathbf{B}_{1}\right) . \quad i=0,1
\end{aligned}
$$

Although there are some minimal discrepancies at the high criteria (likely due to unstable data), the results found when MRI was assessed paralleled those found for marginal $d^{\prime}$ (the bottom four rows of Table 2). That is, when the participants identified the stimulus form, MRI failed in (1) the no-form target conditions for display times of 33 and $83 \mathrm{msec}$, (2) the form-only condition versus the disparate condition for display times of 33 and $83 \mathrm{msec}$, and (3) the form-only condition versus the coincident condition when the display time was $83 \mathrm{msec}$. When the participants identified the stimulus hue, MRI failed only in the hue-only condition versus the coincident condition when the display time was $83 \mathrm{msec}$.

Marginal $\beta$. The marginal $\beta$ analysis does not provide additional information, given the findings from the marginal $d^{\prime}$ and MRI results.

Sampling independence. The analysis of sampling independence assesses whether the probability of reporting both dimensions equals the product of the probabilities of reporting each dimension alone. Sampling independence is sensitive to the covariance between dimensions and is a test of perceptual independence. For sampling independence to hold, the following must be true:

$$
\begin{aligned}
& \left.p\left(a_{i} b_{1} \mid \mathbf{A}_{i} \mathbf{B}_{j}\right)=\left[p_{(} a_{1} b_{0} \mid \mathbf{A}_{i} \mathbf{B}_{j}\right)+p\left(a_{1} b_{1} \mid \mathbf{A}_{i} \mathbf{B}_{j}\right)\right] \\
& \text { * }\left[p\left(a_{0} b_{1} \mid \mathbf{A}_{i} \mathbf{B}_{j}\right)+p\left(a_{1} b_{1} \mid \mathbf{A}_{i} \mathbf{B}_{j}\right)\right] \text {. } \\
& i=0,1 \quad j=0,1
\end{aligned}
$$

Sampling independence held for all conditions except the no-target condition (first four columns of Table 3). In 
the no-target condition, the participants report the presence of both hue and form target feature values more often than would be predicted by sampling independence for all criteria. Because reports in the no-target condition were presumably based on noise in the system, the lack of sampling independence in the no-target condition suggests that the noise in the form stream and that in the hue stream were correlated.

Tetrachoric correlation. Another way to assess perceptual independence is to determine the correlation between participants' ratings of the form dimension and their ratings of the hue dimension for each stimulus. Ashby (1988) showed that sampling independence and uncorrelated ratings are equivalent if (1) decisional separability holds and (2) the participant's response criteria are the same in both tasks. Ashby (1988) also showed that a product moment correlation underestimates the relation between the two dimensions and recommended a tetrachoric correlation as a less biased estimator of the relation between the two dimensions. The tetrachoric correlation is similar to sampling independence in that it divides a distribution into four quadrants and estimates the correlation that results from the proportions in each quadrant assuming a bivariate normal distribution.

When multiple ratings are available, a polychoric correlation using all the ratings is preferred. Ashby (1988) has presented a convenient approximation to the polychoric correlation that is based on the standard tetrachoric method. Specifically, when calculating the tetrachoric correlation, one uses the criterion on each dimension that most closely divides the perceptual distribution in half along that dimension. If participants do not use the form and hue rating scales identically, this approximation of the polychoric correlation is a more sensitive measure than is sampling independence. This approximation is what I used here.

The data show significant correlations for all conditions except the form-only 33 -msec condition and the hue-only 83-msec condition (last three columns of Table 3). All significant correlations were positive, except the form-only 83 -msec condition. Figure 4 contains equal likelihood contours based on the tetrachoric correlations, assuming equal variance.

\section{Regression Model}

To implement the regression model, a regression was run using the following two equations:

$$
\begin{aligned}
f_{i}= & \alpha+\beta{ }_{\mathbf{F}_{1}}\left(\mathbf{F}_{i}\right)+{ }^{\beta} \mathbf{F}_{2}\left(\mathbf{H}_{j}\right)+{ }^{\beta} \mathbf{F}_{3}(\text { Coincident }) \\
& +{ }^{\beta} \mathbf{F}_{4}(\text { Disparate })+e_{\mathbf{F}}
\end{aligned}
$$

and

$$
\begin{aligned}
h_{j}= & \alpha+\beta \mathbf{H}_{1}\left(\mathbf{H}_{j}\right)+\beta{ }_{\mathbf{H}_{2}}\left(\mathbf{F}_{i}\right)+\beta{ }_{\mathbf{H}_{3}}(\text { Coincident }) \\
& +\beta \mathbf{H}_{4}(\text { Disparate })+e_{\mathbf{H}} .
\end{aligned}
$$

Each stimulus variable was dummy coded: 1 if there was a target feature value, 0 if not. Equations $6 \mathrm{a}$ and $6 \mathrm{~b}$ include two interaction terms, each identifying the relative spatial placement of the form and the hue feature values. ${ }^{8}$ If the two target feature values were present and in the same spatial location, the coincident variable was set to 1 ; otherwise, it was set to 0 . If the two target feature values were present and in different spatial locations, the disparate variable was set to 1 ; otherwise, it was set to 0 . Each participant's ratings were standardized (collapsed over stimulus), and the standardized ratings were used as the dependent variable. The model was calculated separately for each participant, and the results were concatenated using the meta-analytic technique described above.

Mean. The average $\beta$ s and the significance for each stimulus variable in the regression model are presented in Table 4. These weights characterize the influence of each stimulus variable on the participants' average response to each target feature value. The regression analysis indicated that the participants' form responses for both display times were influenced by the presence of a hue target. The presence of the hue target increased the participants' ratings on the form dimension for all the stimuli except those in the disparate condition. When the form and the hue target feature values were present in different spatial locations (i.e., the disparate condition), the participants' ratings on the form target feature value decreased below that in the form-only condition. In contrast, the participants' hue responses were not influenced by the presence of the form target feature value, except in the 83 -msec coincident con-

\begin{tabular}{|c|c|c|c|c|c|c|c|c|c|c|}
\hline \multirow{2}{*}{$\begin{array}{l}\text { Time } \\
(\mathrm{msec})\end{array}$} & \multirow[b]{2}{*}{ Intercept } & \multicolumn{9}{|c|}{ Form Response } \\
\hline & & $p$ & $\beta_{\mathbf{F}_{1}}\left(\mathbf{F}_{1}\right)$ & $p$ & $\beta_{\mathbf{F}_{2}}\left(\mathbf{H}_{1}\right)$ & $p$ & $\beta_{\mathbf{F}_{3}}($ Coincident $)$ & $p$ & $\beta_{\mathbf{F}_{4}}$ (Disparate) & $p$ \\
\hline 33 & -.44 & $<.001$ & 1.17 & $<.001$ & 0.12 & $<.001$ & -0.05 & n.s. & -0.20 & $\overline{<.001}$ \\
\hline \multirow[t]{3}{*}{83} & -.43 & $<.001$ & 1.10 & $<.001$ & 0.22 & $<.001$ & -0.09 & .008 & -0.42 & $<.001$ \\
\hline & & \multicolumn{9}{|c|}{ Hue Response } \\
\hline & & $p$ & $\beta_{\mathbf{H}_{1}}\left(\mathbf{H}_{1}\right)$ & $p$ & $\beta_{\mathbf{H}_{2}}\left(\mathbf{F}_{1}\right)$ & $p$ & $\beta_{\mathbf{H}_{3}}($ Coincident $)$ & $p$ & $\beta_{\mathbf{H}_{4}}($ Disparate $)$ & $p$ \\
\hline 33 & -.62 & $<.001$ & 1.66 & $<.001$ & -0.008 & n.s. & 0.03 & n.s. & 0.007 & n.s. \\
\hline 83 & -.68 & $<.001$ & 1.77 & $<.001$ & -0.003 & n.s. & 0.07 & .001 & 0.020 & n.s. \\
\hline
\end{tabular}

Table 4

The Averaged Coefficients of the Regression Equations Described in the Text and Their Significance for Display Times of 33 and $83 \mathrm{msec}$

Note-Perceptual separability is assessed separately for coincident and disparate targets. Perceptual separability fails for a dimension (form or hue) if $\beta_{\cdot_{3}}$ or $\beta_{\cdot_{3}}$ is significant $(p<.01)$. Perceptual separability fails for both coincident and disparate conditions within a dimension if $\beta_{._{2}}$ is significant $(p<.01)$. 
dition. In this condition, the participants' hue ratings increased. The regression findings paralleled the findings of the macroanalysis.

Figure 5 plots the means and equal likelihood contours of the perceptual distributions in two-dimensional GRT space. Again, if perceptual separability holds for a dimension, the bivariate means of the perceptual distributions will fall on the dotted lines perpendicular to that dimension's axis. This condition is free to fail for all distributions except $\mathbf{F}_{0} \mathbf{H}_{0}$. Perceptual independence is represented in Figure 5 by equal likelihood contours based on $\rho_{\mathrm{eFeH}}$, assuming bivariate normal distributions. Perceptual independence holds only if the major and minor axes of the ellipse representing equal likelihood contours are parallel to the hue and form axes.

Variance. To assess whether the variance of participants' responses on one dimension is affected by the level of the feature value of other dimension, tests for homogeneity of variance were run on the standardized ratings of the relevant distributions (see Table 5). The results show that the variances of the 33- and 83-msec coincident stimuli for the form response were significantly smaller than the variances of the form-only target trial for both display times. Similarly, the variance of the coincident stimulus for the hue response was significantly smaller than the variance of the hue-only target for $83 \mathrm{msec}$. There were no other significant differences in variances.

Correlated residuals. To assess perceptual independence, the correlation between the residuals of the two equations was calculated. The results paralleled those of the tetrachoric correlation (see Table 6). The data showed a significant correlation for all the conditions except the form-only 33-msec condition and the hue-only 83-msec condition (see Figure 5).

\section{Discussion}

The regression analysis and Kadlec and Townsend's (1992a, 1992b) micro- and macroanalyses produced parallel results. In particular, the two analyses suggested a perceptual and/or decisional interaction between the form and the hue processing streams. Below, I (1) will compare the results of each analysis, addressing perceptual separability, perceptual independence, and decisional separability, (2) will discuss how these findings relate to the processing model described above, and (3) will discuss the importance of these findings for the validation of the regression model.

\section{Assessing Dimensional Interactions}

Perceptual separability. Two necessary conditions of perceptual separability are (1) $\mu_{\mathbf{A}_{i} \mathbf{B}_{0}}=\mu_{\mathbf{A}_{i} \mathbf{B}_{1}}$, and (2) $\sigma_{\mathbf{A}_{i} \mathbf{B}_{0}}^{2}=\sigma_{\mathbf{A}_{i} \mathbf{B}_{1}}^{2}$. Macroanalysis assesses perceptual separability with marginal $d^{\prime}$ and MRI analyses. The regression model assesses perceptual separability by estimating the effect of stimulus variables on the participants' ratings of the presence of each target feature value. Both analyses produced parallel patterns of results.

\section{$33 \mathrm{msec}$}

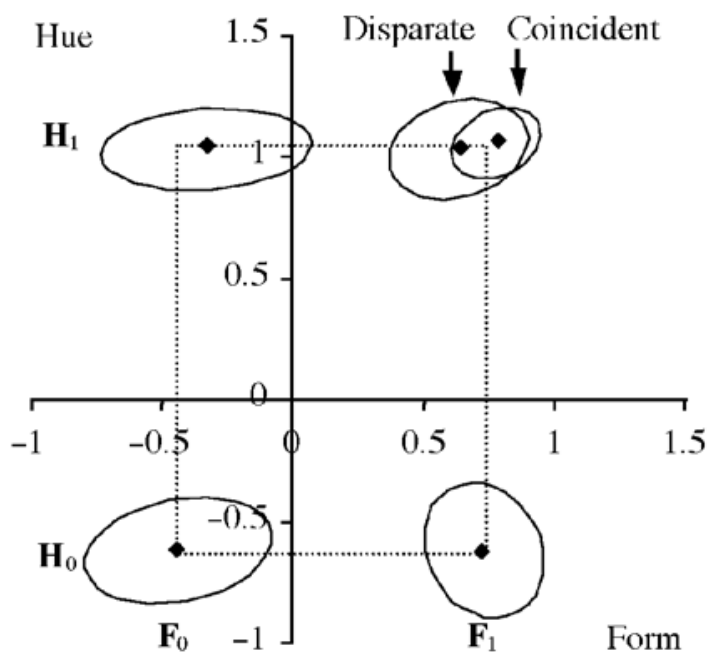

$83 \mathrm{msec}$

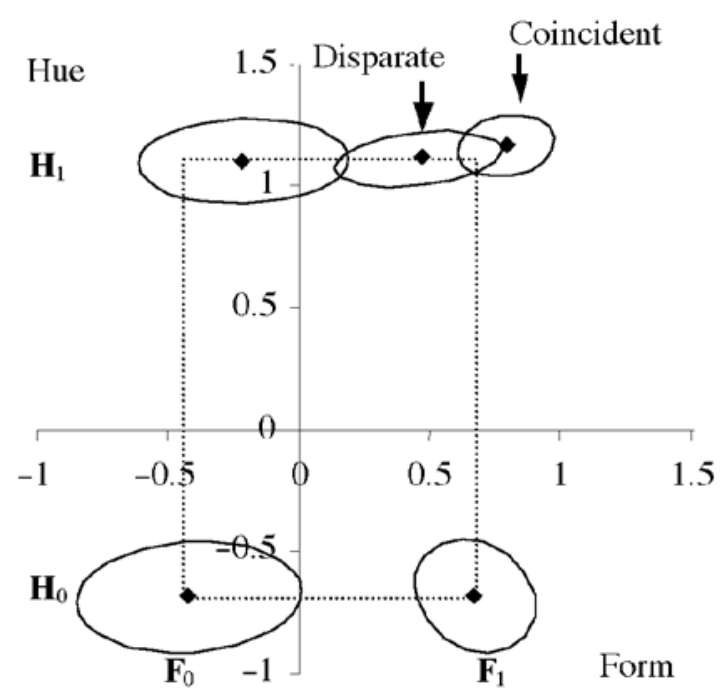

Figure 5. Placement and shapes of the five perceptual distributions associated with the five trial types used in two-dimensional general recognition theory space, based on the regression analysis. The dotted rectangle indicates the predicted position of the bivariate means of the perceptual distributions if hue and form are perceptually separable. The solid ellipses represent equal likelihood contours of the perceptual distributions derived from the results of the product moment correlation analysis and the homogeneity of variance tests, assuming bivariate normal distributions.

Both the regression analysis and the macroanalysis identified identical patterns of data addressing perceptual separability. Specifically, the two analyses showed asymmetric failures of perceptual separability at the 33- and 83-msec display times. The degree and type of perceptual integrality was a function of the relative spatial location of the form and the hue feature values. At 33 and $83 \mathrm{msec}$, detection of the form target feature value was inhibited 
Table 5

The Standard Deviation of Each Response Distribution Along the Hue and Form Axes and Tests of Equality of Relevant Variances (i.e., Form Response, $F_{i} H_{0}=F_{i} H_{1}$; Hue Response, $F_{0} H_{j}=F_{1} H_{j}$ ) for Display Times of 33 and $83 \mathrm{msec}$

\begin{tabular}{|c|c|c|c|c|c|c|c|c|}
\hline \multirow[b]{2}{*}{ Time (msec) } & \multicolumn{8}{|c|}{ Form Response } \\
\hline & $\begin{array}{c}\text { No Target } \\
\left(\mathbf{F}_{0} \mathbf{H}_{0}\right)\end{array}$ & $\begin{array}{c}\text { Hue } \\
\left(\mathbf{F}_{0} \mathbf{H}_{1}\right)\end{array}$ & $p$ & $\begin{array}{c}\text { Form } \\
\left(\mathbf{F}_{1} \mathbf{H}_{0}\right)\end{array}$ & $\begin{array}{c}\text { Disparate } \\
\left(\mathbf{F}_{1} \mathbf{H}_{1}\right)\end{array}$ & $p$ & $\begin{array}{c}\text { Coincident } \\
\left(\mathbf{F}_{1} \mathbf{H}_{1}\right)\end{array}$ & $p$ \\
\hline 33 & .825 & .899 & n.s. & .558 & .612 & n.s. & .413 & $\overline{<.001}$ \\
\hline \multirow[t]{3}{*}{83} & .967 & .892 & n.s. & .546 & .636 & n.s. & .441 & $<.001$ \\
\hline & \multicolumn{8}{|c|}{ Hue Response } \\
\hline & $\begin{array}{c}\text { No Target } \\
\left(\mathbf{F}_{0} \mathbf{H}_{0}\right)\end{array}$ & $\begin{array}{c}\text { Form } \\
\left(\mathbf{F}_{1} \mathbf{H}_{0}\right)\end{array}$ & $p$ & $\begin{array}{c}\text { Hue } \\
\left(\mathbf{F}_{0} \mathbf{H}_{1}\right)\end{array}$ & $\begin{array}{c}\text { Disparate } \\
\left(\mathbf{F}_{1} \mathbf{H}_{1}\right)\end{array}$ & $p$ & $\begin{array}{c}\text { Coincident } \\
\left(\mathbf{F}_{1} \mathbf{H}_{1}\right)\end{array}$ & $p$ \\
\hline 33 & .551 & .571 & n.s. & .415 & .443 & n.s. & .371 & n.s. \\
\hline 83 & .492 & .523 & n.s. & .414 & .349 & n.s. & .265 & $<.001$ \\
\hline
\end{tabular}

Note-Perceptual separability is assessed separately for coincident and disparate targets. Perceptual separability fails for a dimension (form or hue) if the compared measures differ $(p<.01)$.

when the form and the hue target feature values occupied different spatial locations. At $83 \mathrm{msec}$, detection of both the form and the hue target feature values was facilitated when the form and the hue target feature values occupied the same spatial location. This perceptual integrality may be a function of a change in the mean and/or the variance of one or more of the relevant perceptual distributions. Whereas macroanalysis could not adjudicate between these two sources, the regression analysis did. In particular, the results of the regression analysis showed that the improved detection of the form and the hue target feature values in the 83 -msec coincident condition was characterized by a minimal positive mean shift, together with a robust decrease in the variance of the $\mathbf{F}_{1} \mathbf{H}_{1}$ distribution (relative to the $\mathbf{F}_{1} \mathbf{H}_{0}$ and $\mathbf{F}_{0} \mathbf{H}_{1}$ distributions). This finding contrasts with the inhibitory effect present in the disparate condition, which was characterized by a robust negative mean shift and equal variances. These two effects suggest that the perceptual system implements facilitation qualitatively different than it implements inhibition.

Finally, both macroanalysis and regression analysis provided evidence that perceptual integrality increased with stimulus onset asynchrony (SOA). This was demonstrated by the findings that perceptual separability failed on both dimensions for $\mathbf{F}_{1} \mathbf{H}_{1}$ only for the 83-msec display time and that the inhibition of the form dimension in the disparate condition was stronger in the 83 -msec display time than in the 33-msec display time.

Perceptual independence. GRT defines perceptual independence as $\rho_{\mathbf{A B}}=0$. Microanalysis assesses perceptual independence using tests of sampling independence and tetrachoric correlation. The regression model assesses perceptual independence by calculating the correlation between the residuals of the equations predicting participant's responses. Both analyses produced parallel patterns of results.

Although the sampling independence analysis indicated perceptual dependence only in the no-target condition $\left(\mathbf{F}_{0} \mathbf{H}_{0}\right)$, the tetrachoric correlation analysis revealed positive relationships in four of the five perceptual distributions. The lone exception was the correlation between dimensions of the $\mathbf{F}_{1} \mathbf{H}_{0}$ distribution, which was negative. The correlated residuals of the regression analysis showed the same pattern of data and significance values as the tetrachoric correlation. The product moment estimations of the correlations, however, were smaller than the tetrachoric estimations of the correlations. This finding is consistent with that of Ashby (1988), who demonstrated that the product moment correlation underestimates the actual correlation between dimensions. Thus, although the strength of the correlation may be suspect in one or both of these methods, in this instance the direction and pattern of significance were robust across methods.

These results suggest that perceptual independence fails for all five perceptual distributions. The presence of both positive and negative correlations necessitates that any explanation of the interaction between dimensions must be parsimonious with varying directions of correlation. Furthermore, no single transformation that retains the ordinal structure of the data can eliminate both the negative and the positive correlations.

Decisional separability. Decisional separability is characterized by a cognitive decision strategy whereby the participant's judgment of one dimension is independent of his or her judgment of the other dimension. The significantly correlated residuals of the regression analysis give rise to the possibility that decisional separability failed. However, because correlated residuals can arise from Stage 2

Table 6

The Average Correlation and Significance Between Form Response Residuals and Hue Response Residuals for Each Stimulus for Display Times of 33 and $83 \mathrm{msec}$

\begin{tabular}{llccccc}
\hline & & \multicolumn{2}{c}{$33 \mathrm{msec}$} & & \multicolumn{2}{c}{$83 \mathrm{msec}$} \\
\cline { 2 - 4 } \cline { 5 - 6 } \multicolumn{2}{c}{ Stimulus } & $\rho_{\mathrm{eFeH}}$ & $p$ & & $\rho_{\mathrm{eFeH}}$ & $p$ \\
\hline No target & $\left(\mathbf{F}_{0} \mathbf{H}_{0}\right)$ & .15 & $<.001$ & .12 & $<.001$ \\
Form & $\left(\mathbf{F}_{1} \mathbf{H}_{0}\right)$ & -.05 & n.s. & -.12 & $<.001$ \\
Hue & $\left(\mathbf{F}_{0} \mathbf{H}_{1}\right)$ & .14 & $<.001$ & & .04 & n.s. \\
Disparate & $\left(\mathbf{F}_{1} \mathbf{H}_{1}\right)$ & .24 & $<.001$ & & .15 & $<.001$ \\
Coincident & $\left(\mathbf{F}_{1} \mathbf{H}_{1}\right)$ & .31 & $<.001$ & & .13 & $<.001$ \\
\hline
\end{tabular}

Note-Perceptual independence is assessed separately for each stimulus. Perceptual independence fails if $p<.01$. 
and/or 3 interactions, their presence does not guarantee decisional integrality.

Given the pattern of results, neither the regression analysis nor the macro- and microanalyses can differentiate a Stage 2 from a Stage 3 interaction in this data set. Nevertheless, there are several reasons to doubt that the correlated residuals were a result of a Stage 3 interaction. First, the following experimental controls were implemented to encourage decisional separability (see the Perceptual Versus Decisional Effects section): (1) The participants rated each dimension separately, (2) trial type was randomized, (3) order of response was randomized, and (4) the participants were not given accuracy feedback. Although these experimental controls may encourage decisional separability, they do not guarantee it. Second, decisional integrality cannot easily produce the pattern of perceptual integrality present in the data. Specifically, two of the failures of perceptual separability were (1) a negative mean shift on the form dimension in the disparate condition and (2) a positive mean shift on the form dimension in the hue-only and coincident conditions. Although decisional separability may result in a mean shift, it is perplexing why this shift would be positive in the hue-only and coincident conditions and negative in the disparate condition. Such a pattern would require a complex response strategy that is dependent on the participant's knowing which stimulus was presented and then responding contrary to that knowledge in the disparate condition. Such a hypothesis lacks parsimony. Third, a complex and unlikely decision strategy is required to produce both positive and negative dimensional correlations. Finally, the consistency of results across criteria in the SDT analyses suggests parallel response criteria, because criteria that are not parallel have a high probability of producing inconsistent results. Taken as whole, although one cannot rule out decisional interactions, it is more parsimonious to assume that the correlated residuals were the result of a perceptual (Stage 2), not a cognitive (Stage 3), interaction.

Further support for Stage 2 interaction comes from the observation that the perceptual integrality increases with SOA. Recall that the degree of Stage 2 interaction is likely a function of the amount of processing completed before the removal of the stimulus. Therefore, one would expect Stage 2 interactions to increase as SOA increases, at least initially. In contrast, Stage 1 and Stage 3 interactions occur before and after processing, respectively. Consequently, one would not expect Stage 1 and Stage 3 interactions to increase with SOA.

\section{Linking GRT With the Processing Model}

The link between GRT and the processing model necessarily relies on assumptions made in an effort to simplify the problem. One such assumption is that of monoresponse streams. The mono-response stream assumption states that each processing stream provides conscious information about a single dimension. Without the monoresponse stream assumption, researchers are unable to dis- tinguish between multiple streams that each allow a unidimensional response and a single stream that allows multidimensional responses.

The results of both analyses strongly suggest perceptual interaction between the hue and the form dimensions. Specifically, perceptual separability of hue and form generally held when the hue of a stimulus was perceived but failed when the form of a stimulus was perceived. When perceptual separability failed, the data indicate that (1) facilitation between hue and form in the coincident condition involved a reduction of noise, (2) inhibition between hue and form in the disparate condition involved a negative shift in the mean of the perceptual distribution, and (3) the degree of perceptual integrality increased with SOA. The data also indicate the failure of perceptual independence of hue and form for all perceptual distributions. The failure resulted in a positive correlation in all perceptual distributions except the perceptual distribution associated with the form-only target. Because the form and the hue dimensions were correlated in all perceptual distributions and decisional separability likely held, the perceptual integrality revealed by the two analyses may have arisen from an interaction in Stage 1 or 2 of the processing model (see Table 1). To speculate on the source of the perceptual integrality would require additional assumptions.

One such simplifying assumption is that of economy of interactions. The economy of interactions assumption states that because interstream interactions (i.e., Stage 2 interactions) are costly in terms of both processing time and processing resources (Rumelhart, 1989), interstream interactions evolve only when they improve the average performance of the visual system. Without an effect on average performance, interstream interactions have little practical value. The economy of interactions assumption therefore requires a Stage 2 interaction to result in a failure of perceptual separability. Because the data indicate a Stage 2 interaction and the only perceptual integrality present in the data is the influence of the hue value on the perception of form, one must deduce that the hue dimension exerted its influence on the form dimension through the Stage 2 interaction.

Interstream interaction during early vision may be an efficient use of resources. Specifically, the visual system divides the visual field by feature dimension and processes those dimensions in separate neurological streams (for a review, see Fellemen \& Van Essen, 1991; Livingstone \& Hubel, 1984, 1988; Zeki, 1992; Zeki \& Shipp, 1988). This division of labor is more economical than processing the visual scene as a whole (Marr, 1976, 1982; Rumelhart, 1989). However, by dividing the scene, each stream is deprived of the information carried by the other feature dimensions. This will result in no loss of information if the feature dimensions are independent in the environment. However, the spatial position of boundaries around visual objects in different feature dimensions tends to be correlated. To avoid losing this potentially valuable source of 
information, the human visual system may have evolved a mechanism that facilitates the sharing of boundary position information between processing streams (see Cohen, 1997; Kubovy, Cohen, \& Hollier, 1999). Such a system would have the economic advantages of modularity and the accuracy advantages of a unitary system.

\section{The Validity of the Regression Model}

The results of the regression model paralleled those of Kadlec and Townsend's (1992a, 1992b) micro- and macroanalyses. The parallel results suggest that the assumptions associated with the regression model are valid. That is, the response scale adequately measured the perceptual effects of the stimuli, and the participants were able to use that scale appropriately (i.e., the ratings constituted direct estimates). Furthermore, the identification of both perceptual integrality and perceptual dependence indicates that the statistical power associated with the response scale was adequate.

The parallel results of the two analyses in the present experiment suggest that either analysis is appropriate for assessing multidimensionalperceptual space. Each analysis, however, provides slightly different information. For example, Kadlec and Townsend's (1992a, 1992b) analysis assesses the influence of Dimension $\mathbf{A}$ on the perception of Dimension B (i.e., perceptual separability) by assessing the equality of marginal $d^{\prime}$ s and MRI along Dimension B. In contrast, the regression model assesses the same effect by comparing the estimated mean and variance of $\mathbf{A}_{0} \mathbf{B}_{0}$ with that of $\mathbf{A}_{1} \mathbf{B}_{0}$ and the estimated mean and variance of $\mathbf{A}_{0} \mathbf{B}_{1}$ with that of $\mathbf{A}_{1} \mathbf{B}_{1}$ along Dimension $\mathbf{B}$. The differences between these two analyses are subtle yet meaningful. Specifically, Kadlec and Townsend's analysis provides information about a participant's sensitivity to target stimuli, using SDT analysis (i.e., $d_{\mathbf{A}_{0} \mathbf{B}}^{\prime}$ and $d_{\mathbf{A}_{1} \mathbf{B}}^{\prime}$ ). Therefore, the estimated distances carry the information inherent in $d^{\prime}$. However, Kadlec and Townsend's analysis provides little information about the variance of each distribution or the placement of $\mathbf{A}_{0} \mathbf{B}_{0}$ relative to $\mathbf{A}_{1} \mathbf{B}_{0}$ and of $\mathbf{A}_{0} \mathbf{B}_{1}$ relative to $\mathbf{A}_{1} \mathbf{B}_{1}$ along Dimension $\mathbf{B}$. The regression analysis, on the other hand, provides specific information about the variance of each distribution and the placement of $\mathbf{A}_{0} \mathbf{B}_{0}$ relative to $\mathbf{A}_{1} \mathbf{B}_{0}$ and of $\mathbf{A}_{0} \mathbf{B}_{1}$ relative to $\mathbf{A}_{1} \mathbf{B}_{1}$ along Dimension $\mathbf{B}$, but all measured distances are relative and meaningful only within each dimension. For example, one can assess whether a participant is better able to discriminate $\mathbf{A}_{0} \mathbf{B}_{0}$ from $\mathbf{A}_{0} \mathbf{B}_{1}$ or $\mathbf{A}_{1} \mathbf{B}_{0}$ from $\mathbf{A}_{1} \mathbf{B}_{1}$ along Dimension $\mathbf{B}$, but one cannot assess whether one is better able to discriminate $\mathbf{A}_{0} \mathbf{B}_{0}$ from $\mathbf{A}_{0} \mathbf{B}_{1}$ along Dimension $\mathbf{B}$ or $\mathbf{A}_{0} \mathbf{B}_{0}$ from $\mathbf{A}_{1} \mathbf{B}_{0}$ along Dimension $\mathbf{A} .{ }^{9}$ The choice of analysis may therefore depend on the specific information of interest to the researcher.

The present article furthers the research addressing the relation between numerical estimates of perceptual effects and SDT analysis. Specifically, the practical relation between numerical estimates and SDT analysis has been demonstrated in unidimensional space (Braida \& Durlach,
1972; Durlach \& Braida, 1969). In addition, the practical relation between numerical estimates of perceptual effects of specific feature dimensions and SDT analysis has been demonstrated in unidimensional space (Cohen \& Lecci, 2001). The present article extends this literature by demonstrating the practical relation between numerical estimates of the perceptual effects of specific feature dimensions and SDT analysis in multidimensional space.

It should be noted that the regression procedure suffers from the same limitations as all procedures that use numerical estimates as direct perceptual effects. These limitations have been discussed extensively in the context of magnitude estimation (e.g., Gescheider, 1988; Marks \& Algom, 1998; Stevens, 1986) and will, therefore, not be discussed here. Nevertheless, the high concurrent validity between the regression model and Kadlec and Townsend's (1992a, $1992 \mathrm{~b})$ SDT-based procedure suggests that the effects of these limitations were negligible.

\section{Conclusion}

In summary, assessing the perceptual and decisional processes associated with detection in multidimensional space is more complicated than equivalent assessments in unidimensional space (e.g., Ashby \& Townsend, 1986). The procedures developed to assess these processes in multidimensional space all require specialized statistical skills and analysis programs (e.g., Ashby \& Lee, 1991; Kadlec \& Townsend, 1992a, 1992b; Maddox \& Ashby, 1996; Wickens, 1992). The present article has proposed and validated a relatively simple regression procedure that provides information about dimensional interaction similar to that provided by Kadlec and Townsend's macro- and microanalyses. It is hoped that the regression procedure will make multidimensional stimulus detection analysis more available to perceptual researchers.

\section{REFERENCES}

Ashby, F. G. (1988). Estimating the parameters of multidimensional signal detection theory from simultaneous ratings on separate stimulus components. Perception \& Psychophysics, 44, 195-204.

Ashby, F. G. (1989). Stochastic general recognition theory. In D Vickers \& P. I. Smith (Eds.), Human information processing: Measures, mechanisms, and models (pp. 435-457). Amsterdam: Elsevier.

Ashby, F. G., \& LEe, W. W. (1991). Predicting similarity and categorization from identification. Journal of Experimental Psychology: General, 120, 150-172.

Ashby, F. G., \& Maddox, W. T. (1994). A response time theory of separability and integrality in speeded classification. Journal of Mathematical Psychology, 38, 423-466.

Ashby, F. G., \& Townsend, J. T. (1986). Varieties of perceptual independence. Psychological Review, 93, 154-179.

BRAIDA, L. D., \& DurLACH, N. I. (1972). Intensity resolution: II. Resolution in one-interval paradigms. Journal of the Acoustical Society of America, 51, 483-502.

Cohen, D. J. (1997). Visual detection and perceptual independence: Assessing color and form. Perception \& Psychophysics, 59, 623-635.

Conen, D. J., \& LeccI, L. (2001). Using magnitude estimation to investigate the perceptual components of signal detection theory. Psychonomic Bulletin \& Review, 8, 284-293.

Conover, W. J., \& ImAN, R. L. (1981). Rank transformations as a bridge 
between parametric and nonparametric statistics. American Statistician, 35, 124-129.

DeCARLO, L. T. (1998). Signal detection theory and generalized linear models. Psychological Methods, 3, 186-205.

Durlach, N. I., \& BraIDA, L. D. (1969). Intensity perception: I. Preliminary theory of intensity resolution. Journal of the Acoustical Society of America, 46, 372-383.

Dwivedi, T., \& SRIVASTAVA, K. (1978). Optimality of least squares in the seemingly unrelated regressions model. Journal of Econometrics, 7, 391-395.

Felleman, D. J., \& Van Essen, D. C. (1991). Distributed hierarchical processing in the primate cerebral cortex. Cerebral Cortex, 1, 1-47.

GESCHEIDER, G. A. (1988). Psychophysical scaling. Annual Review of Psychology, 39, 169-200.

Green, D. M., \& Swets, J. A. (1966). Signal detection theory and psychophysics. New York: Wiley.

Hirsch, J., Hy lton, R, \& Graham, N. (1982). Simultaneous recognition of two spatial-frequency components. Vision Research, 22, 365-375.

KADLEC, H., \& HicKS, C. L. (1998). Invariance of perceptual space and perceptual separability of stimulus dimensions. Journal of Experimental Psychology: Human Perception \& Performance, 24, 80-104.

KADLEC, H., \& TownSEND, J. T. (1992a). Implications of marginal and conditional detection parameters for the separabilities and independence of perceptual dimensions. Journal of Mathematical Psychology, 36, 325-374.

Kadlec, H., \& Townsend, J. T. (1992b). Signal detection analysis of dimensional interactions. In F. G. Ashby (Ed.), Multidimensional models of perception and cognition (pp. 181-228). Hillsdale, NJ: Erlbaum.

KMENTA, J. (1986). Elements of econometrics (2nd ed.). New York: Macmillan.

Kubovy, M., Cohen, D. J., \& Hollier, J. (1999). Feature integration that routinely occurs without focal attention. Psychonomic Bulletin \& Review, 6, 183-203.

Livingstone, M. S., \& Hubel, D. H. (1984). Anatomy and physiology of the color system in the primate visual cortex. Journal of Neuroscience, 4, 309-356.

Livingstone, M. S., \& Hubel, D. H. (1988). Segregation of form, color, movement, and depth: Anatomy, physiology, and perception. Science, 240, 740-749.

Luce, R. D., \& Krumhansl, C. L. (1988). Measurement, scaling, and psychophysics. In R. C. Atkinson, R. J. Herrnstein, G. Lindzey, \& R. D. Luce (Eds.), Stevens' handbook of experimental psychology: Vol. 1. Perception and motivation (pp. 3-74). New York: Wiley.

Macmillan, N. A., \& Creelman, C. D. (1991). Detection theory: A users guide. New York: Cambridge, University Press.

Maddox, W. T., (2001). Separating perceptual processes from decisional processes in identification and categorization. Perception \& Psychophysics, 63, 1183-1200.

MAdDOx, W. T., \& Ashby,F. G. (1996). Perceptual separability, decisional separability, and the identification-speeded classification task. Journal of Experimental Psychology: Human Perception \& Performance, 22, 795-817.

Maddox, W. T., \& Bogdanov, S. V. (2000). On the relation between decision rules and perceptual representation in multidimensional perceptual categorization. Perception \& Psychophysics, 62, 984-997.

MarKs, L. E., \& Algom, D. (1998). Psychological scaling. In M. H. Birnbaum (Ed.), Measurement, judgment, and decision making (pp. 81178). New York: Academic Press.

Marr, D. (1976). Early processing of visual information. Philosophical Transactions of the Royal Society of London: Series B, 275, 483-524.

MARR, D. (1982). Vision: A computational investigation into the human representation and processing of visual information. San Fransisco: Freeman.

OlzAK, L. A. (1986). Widely separated spatial frequencies: Mechanism interactions. Vision Research, 26, 1143-1153.
Rosenthal, R., \& Rubin, D. B. (1986). Meta-analytic procedures for combining studies with multiple effect sizes. Psychological Bulletin, 99, 400-406.

Rumelhart, D. E. (1989). The architecture of mind: A connectionist approach. In M. I. Posner (Ed.), Foundations of cognitive science (pp. 133160). Cambridge, MA: MIT Press.

Stevens, S. S. (1986). Psychophysics: Introduction to its perceptual, neural, and social prospects. Oxford: Transaction.

STRUBe, M. J. (1985). Combining and comparing significance levels from nonindependenthypothesis tests. Psychological Bulletin, 97, 334-341.

Strube, M. J., \& Miller, R. H. (1986). Comparison of power rates for combined probability procedures: A simulation study. Psychological Bulletin, 99, 407-415.

TANNER, W. P. (1956). Theory of recognition. Journal of the Acoustical Society of America, 28, 882-888.

WiCKENS, T. D. (1992). Maximum-likelihood estimation of a multivariate Gaussian ratings model with excluded data. Journal of Mathematical Psychology, 36, 213-234.

Wickens, T. D., \& OlzaK, L. A. (1992). Three views of association in concurrent detection ratings. In F. G. Ashby (Ed.), Multidimensional models of perception and cognition (pp. 229-252). Hillsdale, NJ: Erlbaum.

ZEKI, S. (1992). The visual image in mind and brain. Scientific American, 267, 69-76.

ZEKI, S., \& SHIPP, S. (1988). The functional logic of cortical connections. Nature, 335, 311-317.

\section{NOTES}

1. Throughout the article, bold uppercase letters (e.g., H) indicate stimulus dimensions (e.g., hue). Lowercase italicized letters (e.g., $h$ ) indicate participants' description of the stimulus dimensions (e.g., hue). Numbers (e.g., 1) indicate a particular value (e.g., blue) of a particular stimulus dimension.

2. Ashby (1989) states that the matrix containing the weights associated with crosstalk between two channels (Stage 2) and those associated with the input lines (Stage 1) should be diagonal for perceptual separability to hold (except in the very unlikely instance in which the weights associated with input lines and those associated with crosstalk cancel each other out).

3. Both Kadlec and Wickens offer their programs to researchers. For a copy of Wickens's program, please e-mail him at twickens@socrates. berkeley.edu. For a copy of Kadlec's program (MSDA), please visit the following Web site: http://web.uvic.ca/psyc/software/.

4. Because a Stage 1 interaction occurs between a single processing stream and the stimulus dimensions, a Stage 1 interaction does not produce dependencies between the participant's responses to each dimension.

5 . Because the computer monitors were not calibrated to ensure isosaturation, the stimuli cannot be considered isosaturation.

6 . To reduce the participants' confusion, the word color, as opposed to hue, was used in the response screen.

7. Over $95 \%$ of the individual participants' results were consistent with the results of the meta-analysis. In particular, approximately $95 \%$ of the individual participants' effects were in the same direction as the significant effects of the meta-analysis, and fewer than $5 \%$ of the individual participants' effects were significant when the meta-analysis showed nonsignificant results.

8. All appropriate coding schemes will result in identical conclusions regarding perceptual separability, perceptual independence, and decisional separability.

9. Inferences about these distances can be made if one assumes that the participants' estimates carry more than ordinal information.

(Manuscript received November 6, 2001; revision accepted for publication March 19, 2003.) 